Research Paper

\title{
Circular RNA CircEZH2 Suppresses Transmissible Gastroenteritis Coronavirus-induced Opening of Mitochondrial Permeability Transition Pore via Targeting MiR-22 in IPEC-J2
}

Xiaomin Zhao*, Xuelian Ma*, Jianxiong Guo, Mi Mi, Kaili Wang, Chuyi Zhang, Xiaoyi Tang, Lingling Chang, Yong Huang, Dewen Tong ${ }^{\circledR}$

College of Veterinary Medicine, Northwest A\&F University, Yangling, Shaanxi 712100, P.R. China

* These authors contributed equally to this work.

$\bowtie$ Corresponding author: Prof. Dewen Tong, Telephone: +86-29-87091622, Fax: +86-29-87091032, E-mail: dwtong@nwsuaf.edu.cn

(c) The author(s). This is an open access article distributed under the terms of the Creative Commons Attribution License (https://creativecommons.org/licenses/by/4.0/). See http://ivyspring.com/terms for full terms and conditions.

Received: 2019.05.09; Accepted: 2019.06.22; Published: 2019.07.25

\begin{abstract}
Transmissible gastroenteritis (TGE) is a contagious and infectious disease that is characterized by severe vomiting and diarrhea of swine, especially piglet, and caused by transmissible gastroenteritis coronavirus (TGEV). TGEV infection provokes mitochondrial damage of porcine intestinal epthelial cell (IPEC), which is responsible for inflammation and cell death. In our previous study, we have demonstrated that circular RNA circEZH2 was down-regulated during TGEV infection and promoted the activation of nuclear factor kappa-light-chain-enhancer of activated B cells (NF-KB) via targeting miR-22 in porcine intestinal epithelial cell line (IPEC-J2). Activation of NF-KB is an important factor for mitochondrial damage. Mitochondrial permeability transition pore (mPTP) opening is a key reason for mitochondrial damage. So, we speculate that circEZH2 may regulate TGEV-induced mPTP opening via NF-kB pathway. In the present study, we found that mPTP opening of IPEC-J2 was occured during TGEV infection and suppressed by circEZH2 via attaching miR-22. Hexokinase 2 (HK2) and interleukin 6 (IL-6) were identified as the targets of miR-22. Silencing HK2 enhanced TGEV-induced mPTP opening, while no effect on NF-KB pathway. Silencing IL-6 promoted TGEV-induced mPTP opening and inhibited NF-KB pathway. Inhibitor of NF-KB increased TGEV-induced $\mathrm{mPTP}$ opening. The data revealed that TGEV-induced $\mathrm{mPTP}$ opening was regulated via two pathways: circEZH2/miR-22/HK2 axis and circEZH2/miR-22/IL-6/NF-KB axis.
\end{abstract}

Key words: transmissible gastroenteritis coronavirus virus, circular RNA, microRNA, mitochondrial permeability transition pore, NF-kB

\section{Introduction}

Coronaviruses (CoVs) are the causative agents of human and a variety of animal diseases. In human, CoVs evoke respiratory tract infection and cause common cold or acute respiratory distress syndrome (ARDS) [1-3]. In animals, CoVs also lead to life-threatening diseases, such as severe enteric and respiratory tract infections, and are economically important pathogens[4-7]. TGEV is a member of Coronaviridae and causes TGE. TGE is a highly contagious gastroenteric disease of swine and results in high mortality of less than 2-week-old piglet. TGEV infection can evoke mitochondrial damage, inflammation, and cell death[8, 9]. TGEV-induced mitochondrial damage is a key factor of inflammation and is caused by abnormal opening of mitochondrial permeability transmission pore (mPTP)[10]. Persistent opening of $\mathrm{mPTP}$ can lead to decrease of the mitochondrial membrane potential $(\triangle \psi \mathrm{m})$, reduction 
of ATP production, release of ROS from mitochondria, finally mitochondrial damage[11-14]. Viral infection is one of the important factors for mitochondrial injury[15-18]. Studies have confirmed that TGEV infection can cause intracellular mitochondrial injury. For example, TGEV infection of IPEC-J2 can cause intracellular mitochondrial autophagy[19]. PK-15 cells infected with TGEV can appear mitochondrial injury, showing an increase of mitochondrial $\mathrm{Ca}^{2+}$ and mitochondrial membrane permeability[20, 21].

miR-22 has a strong correlation with NF- $\mathrm{kB}$ pathway and mitochondrial damage. Recently, it was reported that miR-22 inhibited PI3K/Akt/NF-kB signaling in tongue squamous cell carcinoma (TSCC) cells via targeting KAT6B[22]. miR-22 prevents activation of interferon regulating factor 3 (IRF3) and $\mathrm{NF}-\mathrm{kB}$ pathway[23]. In $\mathrm{H} 9 \mathrm{c} 2$ cardiomyocyte, it was found that miR-22 aggravated H/R-induced mitochondrial damage[24].

Viral infection can regulate NF- $\mathrm{kB}$ and $\mathrm{mPTP}$ opening. Hepatitis $B$ virus $x$ protein $(\mathrm{HBx})$ inhibits $\mathrm{HBV}$-induced apoptosis through activating NF-kB in primary rat hepatocytes. Suppression of $\mathrm{mPTP}$ results in $\mathrm{HBx}$-induced apoptosis, indicating NF- $\mathrm{kB}$ is strongly linked to $\mathrm{mPTP}$ [25]. Activation of NF-KB by TNF-a decreases calcium induced $\mathrm{mPTP}$ opening via repressing ANT1[26]. Recently, we reported that mitochondrial damage was caused by TGEV infection. Moreover, circEZH2 enhanced activation of NF-KB pathway via sponging miR-22 during TGEV-induced inflammation process [21]. circEZH2 is comprised of exon 2 to 8 of $\mathrm{EZH} 2$ (ENSSSCG00000028840) and is derived from 109, 411, $982 \mathrm{bp}-109,431,724 \mathrm{bp}$ region of the sense strand of Chromosome 9. NF-kB pathway is an important pathway that related to regulating mitochondrial damage and inflammation[27]. So, we propose a hypothesis that circEZH2 may regulate $\mathrm{mPTP}$ opening via miR-22.

To prove the hypothesis, we investigated the effects of circEZH2 and miR-22 on TGEV-induced $\mathrm{mPTP}$ opening and NF-kB pathway, identified the targets of miR-22, and tested the effects of miR-22 targets on mPTP opening and NF-kB pathway. The data demonstrated that circEZH2 attenuated TGEV-induced mPTP opening via attaching miR-22. miR-22 promoted mPTP opening by targeting HK2 and IL-6. Moreover, IL-6 inhibited mPTP opening by activating NF-KB pathway, while HK2 had no significant impact on activation of NF-KB pathway. The data will provide a potential pharmacologic target for prevention and control of TGE.

\section{Materials and Methods}

\section{Antibodies, cells, and virus}

$\beta$-actin monoclonal antibody was purchased from Santa Cruz (US). Horseradish peroxidase (HRP)-conjugated secondary antibody was purchased from Pierce (US). Hexokinase II (HK2) and Phospho-NF-kB p65 (p-p65) Rabbit monoclonal antibody was purchased from Cell Signaling Technology (US). Dylight594-conjugated secondary antibody was purchased from Genshare Biological (CHN). IPEC-J2 cell line was gifted from Zhanyong Wei (Henan Agricultural University, CHN). Cells were cultured in Dulbecco's Modified Eagle Medium (DMEM)/F-12/HAM (Thermo Fisher Scientific, US), which was supplemented with $100 \mathrm{IU}$ of penicillin and $100 \mathrm{mg}$ of streptomycin per $\mathrm{ml}$, at $37^{\circ} \mathrm{C}$ in an incubator with $5 \% \mathrm{CO}_{2}$. The TGEV Shaanxi strain was separated from TGEV-infected piglets.

\section{Animal experiments}

Six new-born piglets from the same sow, which are free of TGEV, porcine epidemic diarrhea virus (PEDV), Porcine Rotavirus (PoRV), and porcine circovirus type 2 (PCV2), were randomly assigned to 2 groups, 3 piglets per group, and housed separately. Group 1 was orally infected with $5 \mathrm{ml}$ phosphate-buffered saline (PBS), named Mock group as the uninfected control. Group 2 piglets were orally infected with $5 \mathrm{ml}$ of $10^{7}$ median (50\%) tissue culture infective doses (TCID50)/ $\mathrm{ml}$ of TGEV as experimental group. All piglets were euthanized at $48 \mathrm{~h}$ after diarrhea and the jejunum tissues were immediately sampled.

\section{Histological analysis}

Jejunum tissue were fixed in $4 \%$ paraformaldehyde for $48 \mathrm{~h}$ at room temperature, embedded in paraffin, and sliced. The morphological observation of porcine jejunum was conducted after hematoxylin-eosin (HE) staining using Aperio Digital Pathology Slide Scanners (Leica, GER).

\section{Transmission electron microscopy (TEM)}

The jejunum tissue samples were cut into smaller pieces $(1 \mathrm{~mm} \times 1 \mathrm{~mm} \times 3 \mathrm{~mm})$. The monolayer of IPEC-J2 cells infected with TGEV or Mock were fixed in situ for $24 \mathrm{~h}$ with a mixture of $2.5 \%$ glutaraldehyde and $2 \%$ formaldehyde in PBS ( $\mathrm{pH} 7.2$ ) for $6 \mathrm{~h}$ at room temperature. The samples were removed from the dishes and transferred to Eppendorf tubes. After centrifugation in a microcentrifuge at $300 \mathrm{~g}$ for $5 \mathrm{~min}$, the samples were washed with PBS and postfixed with a mixture of $1 \%$ osmic acid in distilled water for $4 \mathrm{~h}$ at $4^{\circ} \mathrm{C}$. After washing four times with PBS, 
samples were dehydrated with graded ethanol $(30,50$, 70,90 , and $100 \%$ ) for $10 \mathrm{~min}$ each concentration at room temperature. The tissues were sequentially infiltrated in LR White Resin (Sigma-Aldrich, US) and ethanol (1:3) for $2 \mathrm{~h}$ at room temperature, in the LR White Resin and ethanol (1:1) for $8 \mathrm{~h}$ at room temperature, in LR White Resin and ethanol (3:1) for $24 \mathrm{~h}$ at room temperature, and in LR White Resin for 2 days at room temperature. Ultrathin (75-nm-thick) sections of the samples were stained with both uranyl acetate and lead citrate by standard procedures. All sections were observed and photographed with a FEI Tecnai G2 Spirit Bio-TWIN transmission electron microscope (FEI, US).

\section{Mitochondrial Permeability Transition Pore (mPTP) activity}

Cells were infected with TGEV at 1.0 MOI for 24 h. Meanwhile, the mock infection was carried out. Each sample was treated with $5 \mu \mathrm{l}$ of BBcellProbe ${ }^{\mathrm{TM}}$ M61 probe $(200 \mu \mathrm{M})$ (BestBio, Shanghai, $\mathrm{CHN})$ for 5 min and quencher for $15 \mathrm{~min}$ at $37^{\circ} \mathrm{C}$. The fluorescence was measured by flow cytometry, with 15,000 events collecting. Opening of $\mathrm{mPTP}$ results in a decrease of fluorescence.

\section{Fluorescence in situ hybridization (FISH)}

Specific probes to circEZH2 sequence and miR-22 were synthesized by Genepharma (Shanghai, $\mathrm{CHN}$ ) and used in in situ hybridization (The sequences are shown in Table S3). In brief, CY3-labelled probe was specific to circEZH2. FAM-labelled probe was specific to miR-22. Nuclei were counterstained with 4,6-diamidino-2phenylindole (DAPI). All the procedures were conducted according to the manufactory's instruction (Genepharma, Shanghai, CHN). Specimens were analyzed using LEICA TCS SP8 confocal microscopy (Leica, GER).

\section{RNA extraction}

The IPEC-J2 cells were infected with TGEV (MOI=1.0) when $70 \%$ confluent in the culture dish and harvested at $24 \mathrm{~h}$ post infection (hpi). The villus of jejunum was scraped and immediately frozen in liquid nitrogen for RNA extraction. RNA was extracted using Trizol reagent (Invitrogen, US) according to the manufacturer's instructions. RNA concentration and quality were measured by NanoDrop1000 spectrophotometer (NanoDrop Technologies, US).

\section{Quantification of miRNAs, circRNAs, and mRNAs by qRT-PCR}

The total RNA was reversely transcribed by M-MLV reverse transcriptase (Invitrogen, US).
Especially, for reverse transcription of circRNAs, the total RNA was treated with RNase R (Epicentre, US) to remove liner RNA and then was subjected to reverse transcription. qRT-PCR was performed as described previously on iQ5 real-time PCR System (Bio-Rad, US) [28]. The relative quantification of miRNAs was normalized to U6 using $2^{-\Delta \Delta C t}$ method. The relative quantification of mRNAs and circRNAs were normalized to $\beta$-actin using the $2^{-\Delta \Delta \mathrm{Ct}}$ method (The primers are shown in Table S4).

\section{Prediction and analysis of $\mathbf{m i R}-22$ targets}

RNAhybrid (v2.1.2) + svm_light (v6.01), Miranda (v3.3a) and TargetScan (V7.0) were used to predict the target genes of miR-22. A intersection between miR-22 targets and identified genes by sequencing was obtained and presented by Venn diagrams. GO term analysis of the target genes were performed by DAVID Bioinformatics Resources 6.8 (https://david.ncifcrf.gov/home.jsp). The miR-22mRNA regulatory network was constructed by Cytoscape (v3.5.1) (http://www.cytoscape.org/).

\section{Luciferase reporter assay}

3' UTRs of candidate target genes containing the binding site of miR-22 were respectively amplified by PCR and were cloned into the vector psiCHECK-2 (Promega, US). To obtain mutation of miR-22 complementary sites within the $3^{\prime}$ UTR of HK2 and IL-6, the binding sites of miR-22 seed region in $3^{\prime}$ UTRs were mutated following a mutagenesis protocol [29] (The primers are shown in Table S4). The miR-22 mimics, miR-22 mimics control, miR-22 inhibitor, and miR-22 inhibitor control were designed and synthesized by Genepharma (Shanghai, CHN) (The sequences are shown in Table S3). For the luciferase reporter assay, IPEC-J2 cells were seeded in 24-well plate and then co-transfected with $100 \mathrm{ng}$ plasmid and $100 \mathrm{nM}$ of miR-22 mimics, or miR-22 inhibitor, or control, using Lipofectamine 3000 (Invitrogen, US) according to the manufacturer's instructions. At $48 \mathrm{~h}$ post transfection (hpt), the luciferase activities were measured using Dual-Glo Luciferase Assay System (Promega, US) following the manufacturer's manual.

\section{Analysis of Protein-Protein interactions}

HK2 was searched against STRING database (version 10.0) for protein-protein interactions (https://string-db.org/cgi/input.pl).

\section{Construction of recombinant plasmid for circEZH2 overexpression}

The full-length cDNA of circEZH2 was synthesized by Genebio (Shanghai, CHN) and cloned into the pcircRNA between two frames. The recombinant plasmid was named pcircRNA- 
circEZH2.

\section{Transfection of siRNAs and plasmids}

IPEC-J2 cells were maintained in a 6-well plate with DF-12 medium supplemented with 10\% FBS and not collected until 50-70\% confluent. Cells were transfected with siRNAs (Shanghai, CHN) (The sequences are shown in Table S3), or plasmids using Lipofectamine 3000 (Invitrogen, US) according to the manufacturer's instructions. Countermeasure and overexpression effect was examined by RT-qPCR using RNA extracted 48 hours after transfection.

\section{Western blot analysis}

Cells were treated with RIPA lysis buffer containing phenylmethyl sulonylfluoride (PMSF). Protein concentration was determined using BCA Protein Assay Regent (Pierce, US). Proteins were separated using sodium dodecyl sulfatepolyacrylamide gel elecrophoresis (SDS-PAGE) and subsequently transferred onto polyvinylidene difluoride (PVDF) membrane (Millipore Corp, US). The PVDF membrane was blocked with $5 \%$ non-fat dry milk for $2 \mathrm{~h}$ at room temperature and then incubated with primary antibodies overnight at $4{ }^{\circ} \mathrm{C}$. Subsequently, the membrane was incubated with HRP-conjugated secondary antibody at room temperature for $1 \mathrm{~h}$. The signal was detected with enhanced chemiluminescence (ECL) kit (Genshare, CHN).

\section{Statistics}

The data was presented as the means \pm SEM. Statistical significance was analyzed by unpaired Student's t-test, where ${ }^{*} p<0.05,{ }^{* *} p<0.01$.

\section{Results}

\section{TGEV induced opening of $\mathrm{mPTP}$}

The jejunum of the piglets were fixed, embedded, sliced, and stained using HE. The sections were observed using light microscope. The results showed that the jejunum villi of mock-infected piglets were long and slender (Figure 1A), while the villi of TGEV-infected piglets were shortened and fell away (Figure 1B). Ultrastructural changes were observed using transmission electron microscopy. The results showed virus particles appeared in the cytoplasmic matrix and mitochondria swelling was found in TGEV infected group (Figure $1 \mathrm{C}$ to $\mathrm{F}$ ). The fluorogenic probe, BBcell ProbeTM M61, was used to evaluate the effect of TGEV on $\mathrm{MPTP}$ opening in IPEC-J2. These results indicated that the fluorescent value of TGEV-infected cells showed a significant decrease in comparison with the mock-infected cells (Figure $1 \mathrm{G}$ and $\mathrm{H}$ ).

\section{circEZH2 suppressed $\mathrm{MPTP}$ opening induced by TGEV infection}

Our previous circRNAs sequencing date (available in the Sequence Read Archive with accession number SRP128150) demonstrated that some circRNAs differentially expressed during TGEV infection in IPEC-J2. The circular RNA ssc_circ_009380 was differentially down-regulated during TGEV infection[21]. Analysis of ssc_circ_009380 sequence showed that it was comprised of exon 2 to 8 of EZH2 (ENSSSCG00000028840) that derived from 109, 411, 982-109, 431, $724 \mathrm{bp}$ regions of the sense strand of Chromosome 9 (Figure 2A). Therefore, circular RNA ssc_circ_009380 was named "circEZH2". To confirm the circular characteristics of circEZH2, we next digested total RNA with or without RNase R. Compared to the linear $\beta$-actin mRNAs, circEZH2 was obviously resistant to Rnase $\mathrm{R}$ (Figure 2B). In addition, we found circEZH2 was localized at both nuclear and cytoplasm (Figure 2C and D). Meanwhile, The levels of circEZH2 were measured by qRT-PCR and FISH. The results revealed that circEZH2 was down-regulated during TGEV infection in vitro (Figure 2E and Figure S1) and in vivo (Figure 2F and Figure S1), which is correlated with the Solexa high-throughput sequencing. To investigate the impact of circEZH2 on MPTP opening induced by TGEV, IPEC-J2 cells were transfected with pcircRNA-circEZH2 (or pcircRNA, si-circEZH2, NC siRNA) and subsequently infected with TGEV at 1 MOI for $24 \mathrm{~h}$. The circEZH2 level was significantly increased by pcircRNA-circEZH2 and remarkably decreased by si-circEZH2 (Figure $2 \mathrm{G}$ and $\mathrm{H}$ ). The level of MPTP opening was tested using BBcellProbeTM M61 probe. These results suggested fluorescence intensity of cells infected with TGEV significantly increased by pcircRNA-circEZH2 and significantly decreased by si-circEZH2 in comparison with negative control (Figure 2I to K).

\section{miR-22 promoted MPTP opening induced by TGEV infection.}

The secondary structure of pre-miR-22 was predicted using RNAstructure version 6.0.1 and shown in Figure S2A. The FISH analysis showed that miR-22 distributed in cytoplasm of cells in vitro and in vivo (Figure 3A). Meanwhile, the level of miR-22 in IPEC-J2 and villi of jejunum that were infected with TGEV or Mock infection was evaluated by qRT-PCR and FISH. miR-22 significantly increased both in vitro (Figure 3B and Figure S2B) and in vivo (Figure 3C and Figure S2B) during TGEV infection. To investigate the impact of miR-22 on mPTP opening induced by TGEV infection, IPEC-J2 cells were respectively transfected 
with miR-22 mimics (or miRNA mimics control, miR-22 inhibitor, miRNA inhibitor control) and subsequently infected with TGEV at 1 MOI for $24 \mathrm{~h}$. The miR-22 level was significantly increased by miR-22 mimics and remarkably decreased by miR-22 inhibitor (Figure 3D and E). The level of $\mathrm{mPTP}$ opening was increased by miR-22 mimics and significantly decreased by miR-22 inhibitor (Figure 3F to $\mathrm{H})$.

\section{circEZH2 suppressed $\mathrm{MPTP}$ opening and promoted NF-KB pathway via binding miR-22}

We previously identified that miR-22 could directly bind to circEZH2 via dual-luciferase reporter assay[21]. To provide further evidence on circEZH2 functions as miR-22 sponge, FISH assay was conducted. Co-localization of circEZH 2 and miR-22 was observed in the cytoplasm of IPEC-J2 cells, indicating circEZH2 interacts with miR-22 (Figure $4 \mathrm{~A})$. To examine whether circEZH2 suppressed $\mathrm{mPTP}$ opening via interacting with miR-22, miR-22 mimics and pcircRNA-circEZH2 were co-transfected into IPEC-J2 cells. The fluorescence intensity was significantly inhibited by miR-22 mimics and pcircRNA-circEZH2 co-transfection compared with pcircRNA-circEZH2 alone (Figure 4B and C). Previous studies have suggested that circEZH2 promoted protein level of p-ІкB- $\alpha$ and accumulation of p65 in nucleus in TGEV-infected IPEC-J2 cells[21]. Further analysis showed that TGEV induced accumulation of p-p-65, and miR-22 inhibited this process (Figure 4D and E). The protein level of p-p65 significantly decreased in IPEC-J2 cells co-transfected with miR-22 mimics and pcircRNA-circEZH2 compared with in cells transfected with pcircRNA-circEZH2 alone (Figure 4F). The data suggested that circEZH2 could promote NF-kB pathway and suppress $\mathrm{mPTP}$ opening by binding miR-22.
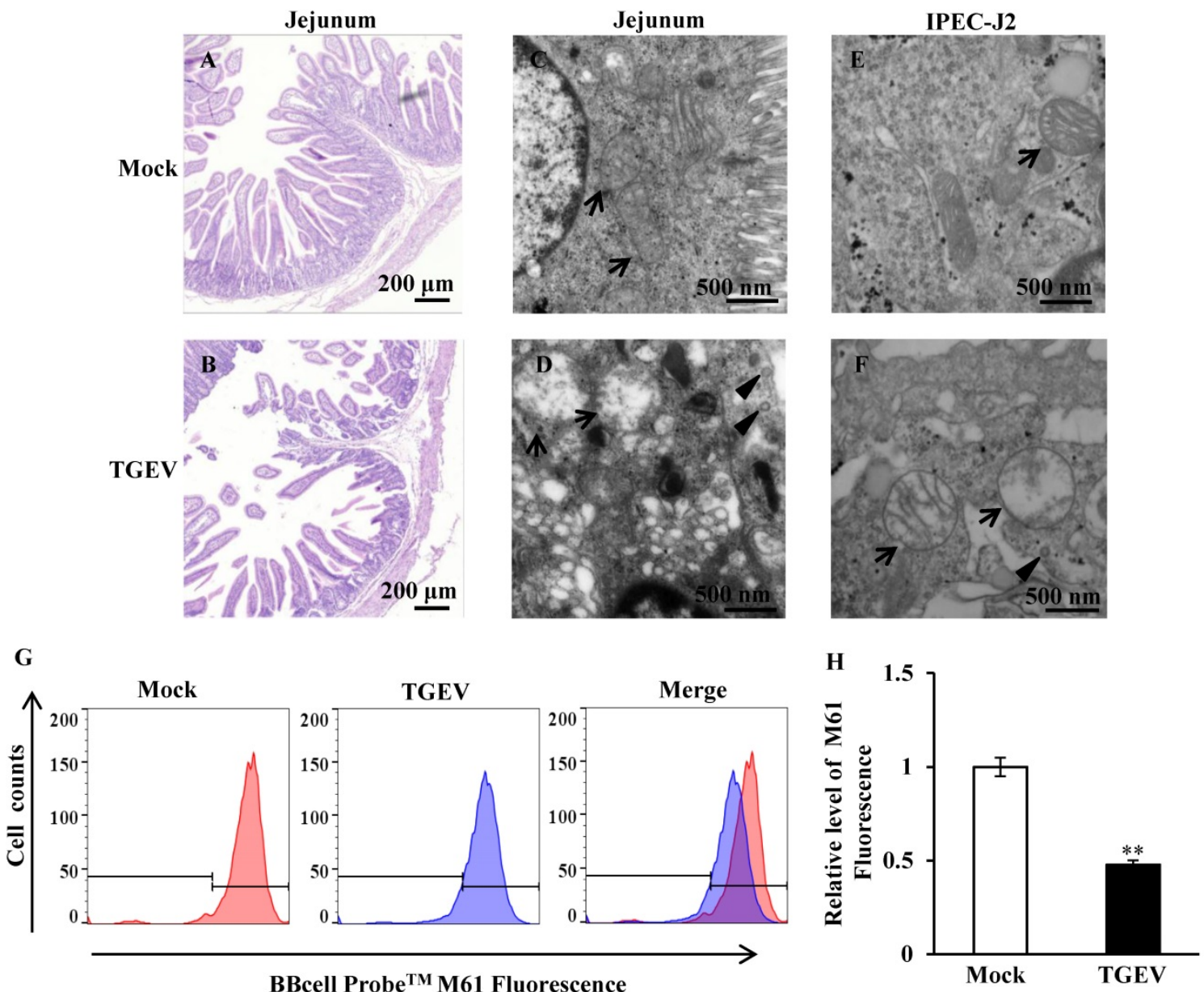

Figure 1 TGEV infection resulted in mitochondrial damage and $\mathbf{m P T P}$ opening. (A) and (B) Histological changes of piglet jejunum infected with TGEV. (C) and (D) Ultrastructure changes of mitochondria in jejunum IPEC of piglet in response to TGEV infection. (E) and (F) Ultrastructure changes of mitochondria in IPEC-J2 in response to TGEV infection. Black arrows indicate mitochondrion. Black triangles indicate viral particle. $(\mathrm{G})$ and $(\mathrm{H})$ The degree of mPTP opening of IPEC-J2 infected with TGEV. The fluorescence was measured via FCM and quantified with Fluorescence Activated Cell Sorting (FACS). $* * p<0.01$. 
circEZH2 Sanger Sequencing

E

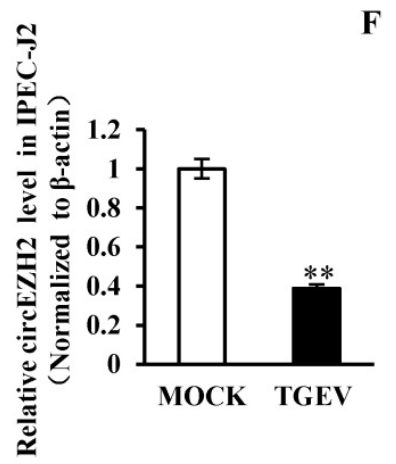

A

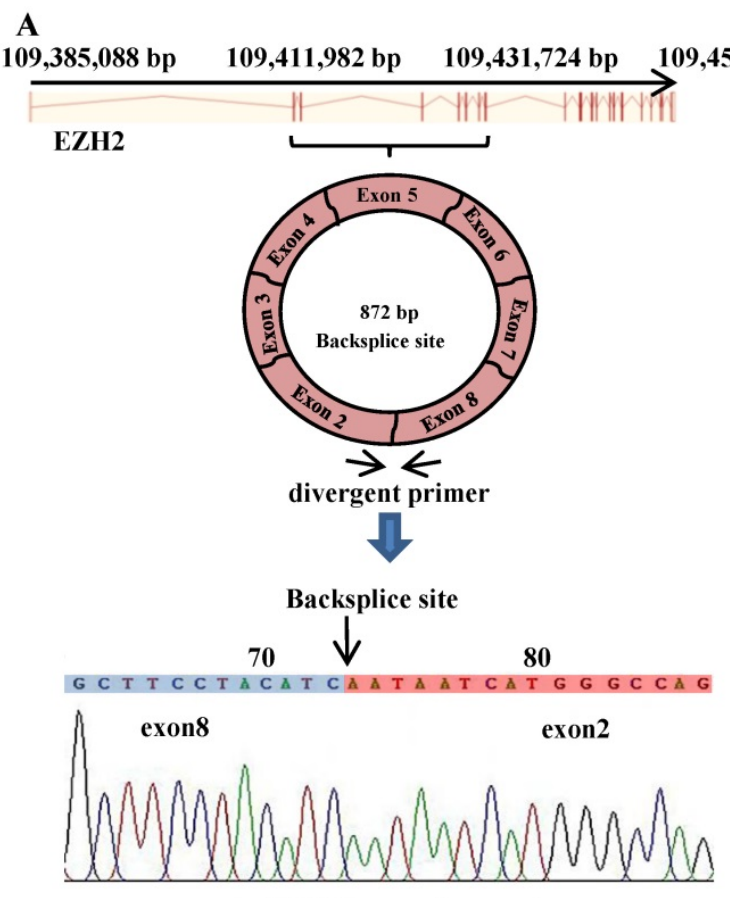

divergent primer

Backsplice site

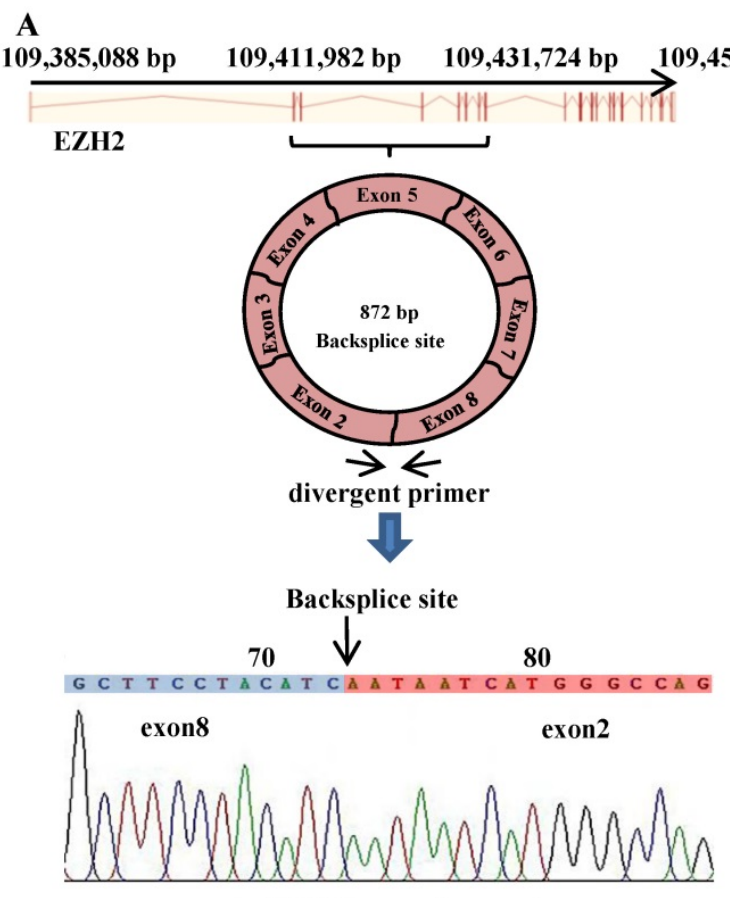

GCT TCCTACATCA A A ATCATGGGCCAG

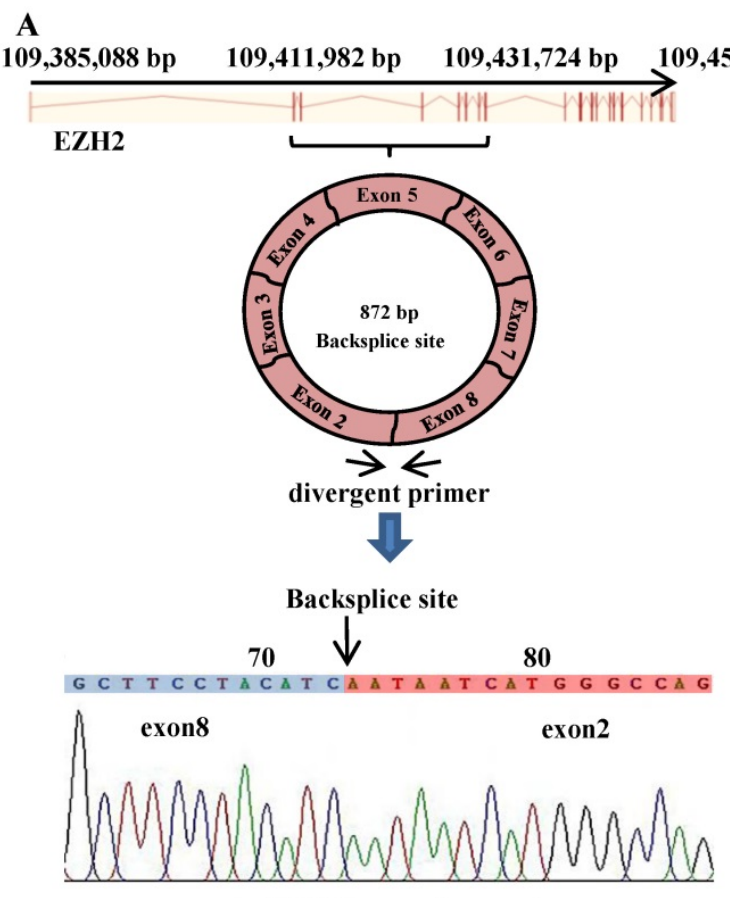

B

$$
\text { bp }
$$

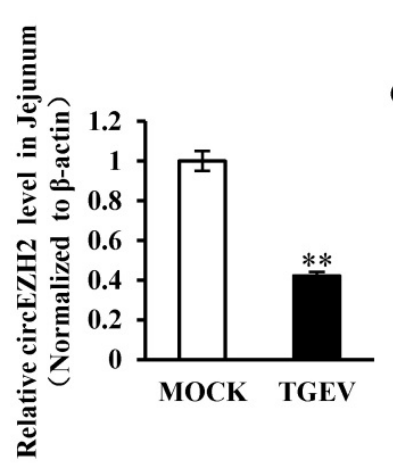

1.0 MOI TGEV 24 hpi

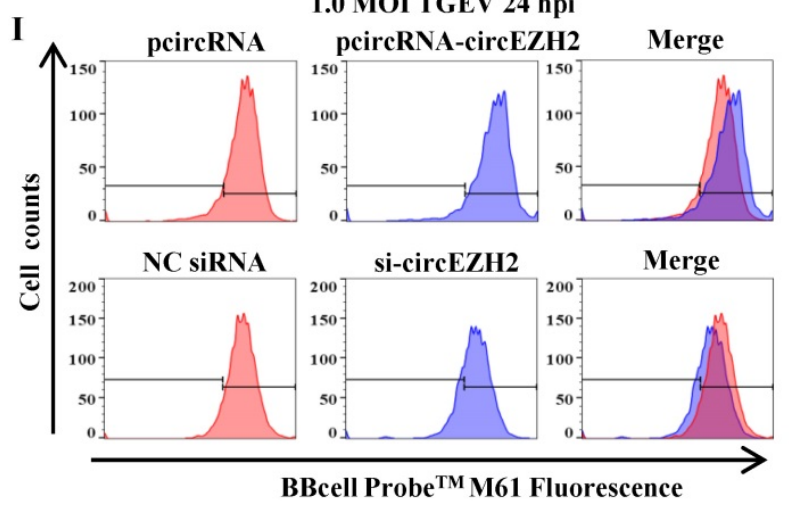

D

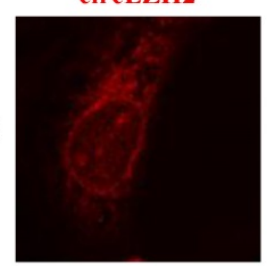

Jejunum

口RNase R(-)

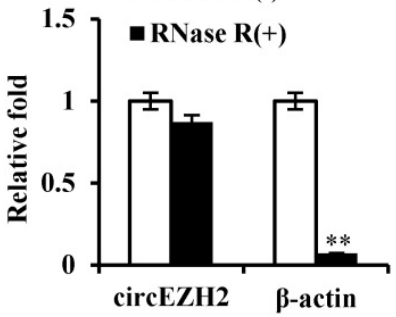

IPEC-J2

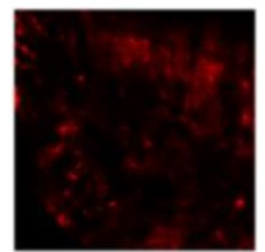

G

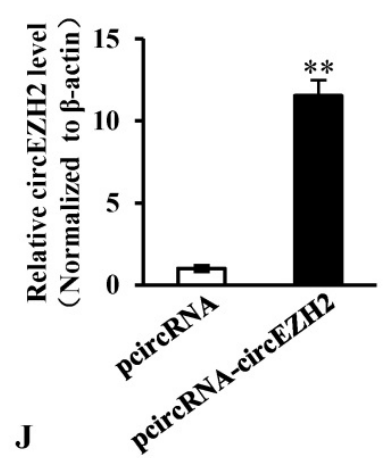

H

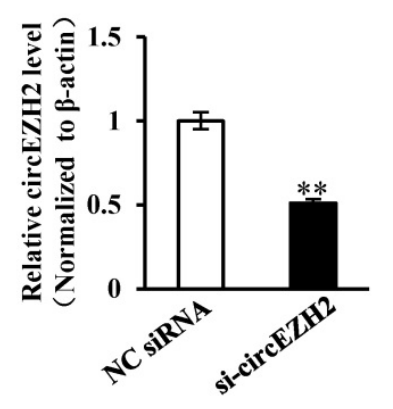

K

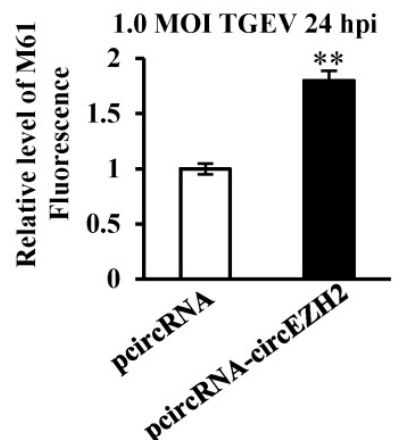

DAPI
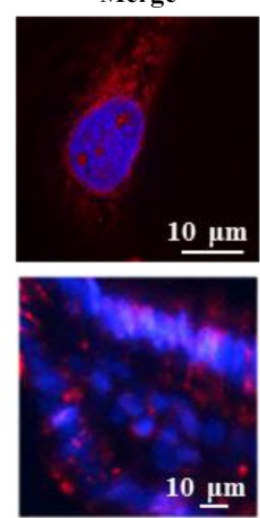

$0 \underline{\mu m}$

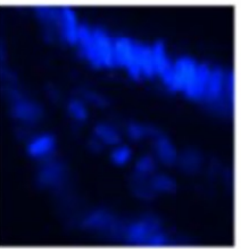

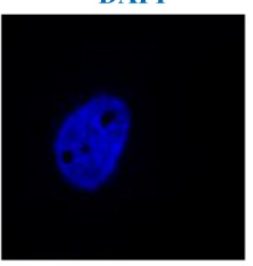

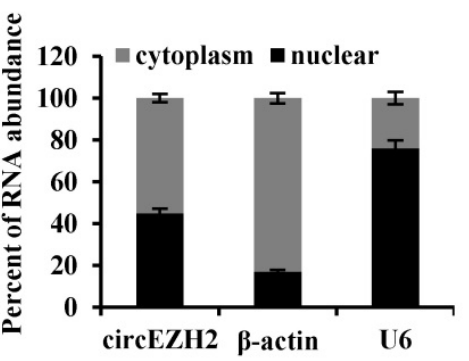

Figure 2 circEZH2 localized at both nucleus and cytoplasm, and suppressed TGEV-induced mPTP opening. (A) The genomic loci of circEZH2 and the Sanger sequence of backsplice site of circEZH2. Arrows represent divergent primers binding to the genome region of circEZH2. (B) $q R T-P C R$ analysis of $\beta$-actin and circEZH2 after Rnase R treatment. (C) qRT-PCR analysis of circEZH2 in cytoplasm and nuclear. mRNA level of $\beta$-actin in cytoplasm and U6 level in nuclear fraction were respectively referred as quality controls of cytoplasm and nuclear fractions. (D) circEZH2 was localized at both nucleus and cytoplasm in IPEC-J2 and jejunum IPEC of piglet. (E) Fold change of circEZH2 level in IPEC-J2 in response to TGEV infection. (F) Fold change of circEZH2 level in jejunum IPEC of piglet in response to TGEV infection. (G) Overexpression effect of pcircRNA-circEZH2. (H) Silencing effect of si-circEZH2. (I) The effect of circEZH2 on TGEV-induced mPTP opening in IPEC-J2. (J) and (K) Quantification of mPTP opening level of Figure 21. ** $p<0.01$. 
A

miR-22

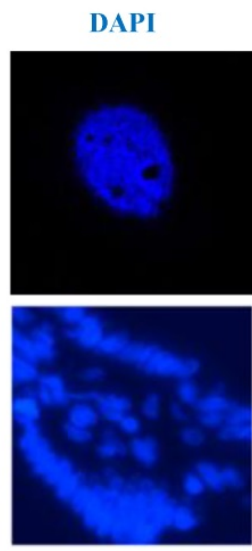

1.0 MOI TG EV 24 hpi

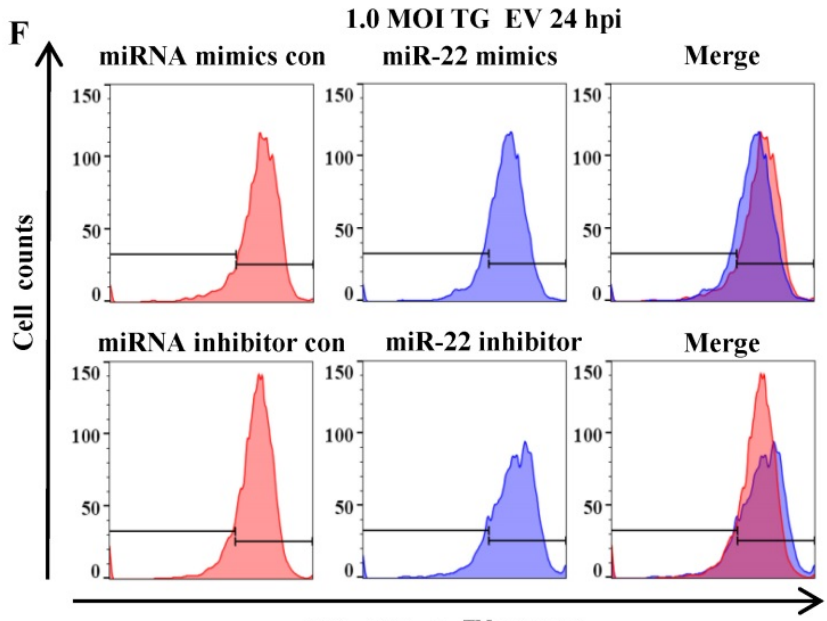

BBcell Probe ${ }^{\mathrm{TM}}$ M61 Fluorescence

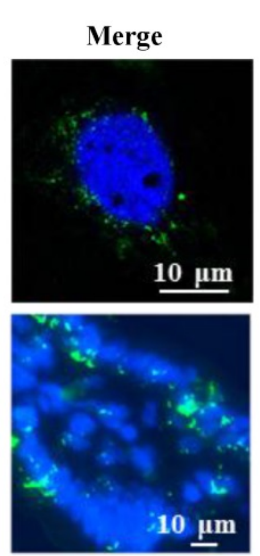

B

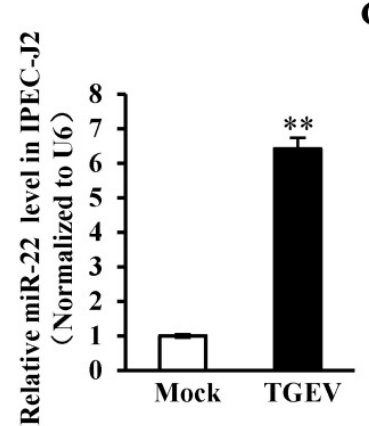

D

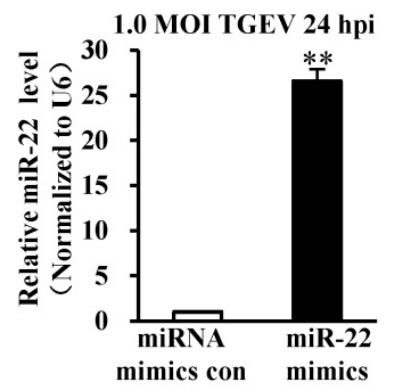

G

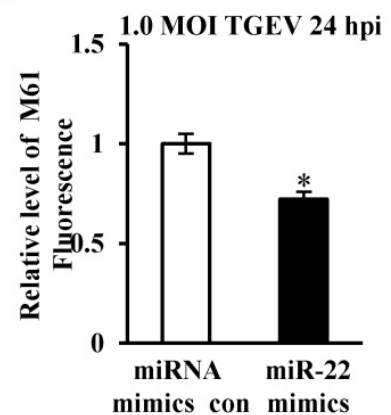

C

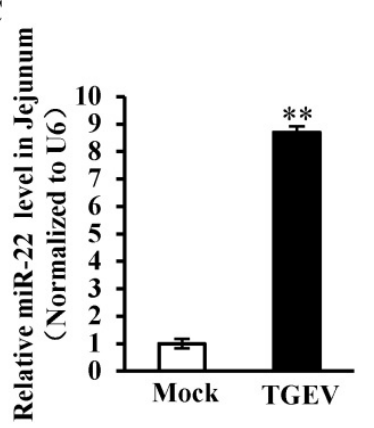

E

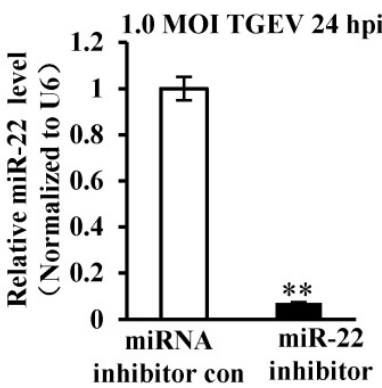

H

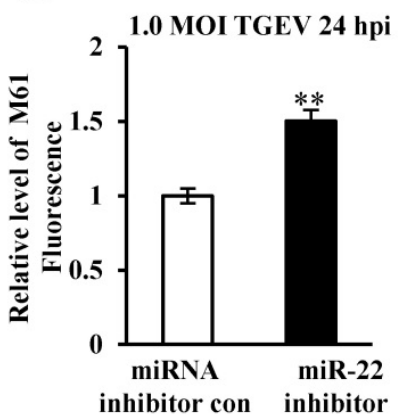

Figure 3 miR-22 was localized at cytoplasm and promoted mPTP opening. (A) Localization of miR-22 in IPEC-J2 and jejunum IPEC of piglet. (B) Fold change of miR-22 in IPEC-J2 in response to TGEV infection. (C) Fold change of miR-22 in jejunum IPEC of piglet in response to TGEV infection. (D) Overexpression effect of miR-22 mimics. (E) Silencing effect of miR-22 inhibitor. (F) The effect of miR-22 on TGEV-induced mPTP opening in IPEC-J2. (G) and (H) Quantification of mPTP opening level of Figure 3F. * $p<0.05$ and $*^{*} p<0.01$ in comparison with the control.

\section{Analysis of miR-22 targets and mRNAs}

The intersection of identified mRNAs of each sample by sequencing was obtained and presented via Venn diagram. 8214 intersective mRNAs were obtained (Table S1 and Figure 5A). 378 target genes of miR-22 were predicted and intersected with the 8214 mRNAs, 197 genes were intersected (Table S2 and Figure 5B). GO enrichment analysis of the 197 genes was performed and presented (Table S3). The result showed that 30 target genes were localized at mitochondria or participated in the immune system process (Figure 5C and D). To identify the targets, 16 target genes of miR-22 that related to mitochondrion and inflammation were selected for identification. The 3' UTR of the 16 target genes were cloned into 3' UTR of dual-luciferase reporter plasmid to gain the recombinant plasmid. The luciferase activities were detected. The results showed that luciferase activity of plasmid containing the 3' UTR of HK2 was markedly lower than that of other targets (Figure 5E). To further identify the direct binding between miR-22 and $3^{\prime}$ UTR of HK2 mRNA, the binding site of miR-22 seed sequence at HK2 3' UTR were mutated with the 4-bp substitution (Figure 5F). The constructs and miR-22 mimics (or miRNA mimics control, miR-22 inhibitor, miRNA inhibitor control) were co-transfected into IPEC-J2 cells. The luciferase activities were measured. Compared with the control, introduction of exogenous miR-22 decreased the psi-HK2-WT reporter activity by $45 \%$, while over-expression and inhibition of miR-22 did not affect luciferase activity (Figure 5G and $\mathrm{H}$ ). The effects of miR-22 on transcription and expression of HK2 were tested using real-time PCR and western blot. The results indicated that the transcriptional level of HK2 was not influenced by miR-22 mimics and inhibitor (Figure 
5I). However, the protein level of HK2 was down-regulated by miR-22 mimics and up-regulated by miR-22 inhibitor (Figure 5J). Together, our data conclusively demonstrated that HK2 is a direct target of miR-22 in IPEC-J2 cells.

\section{si-HK2 promoted MPTP opening induced by TGEV in IPEC-J2}

HK2 was introduced into the web-tool STRING to generate protein-protein interaction networks. We found that VDAC1, an important protein in $\mathrm{MPTP}$, might interact with HK2 (Figure 6A). IPEC-J2 cells were transfected with si-HK2 or Negative Control of siRNA (NC siRNA), and subsequently infected with TGEV at $1 \mathrm{MOI}$ for $24 \mathrm{~h}$. The HK2 levels on both mRNA and protein were significantly inhibited by si-HK2 (Figure 6B and C). The fluorescence intensity for BBcellProbe ${ }^{\mathrm{TM}} \mathrm{M} 61$ was measured using flow cytometry and markedly suppressed by si-HK2 (Figure 6D and E).
A

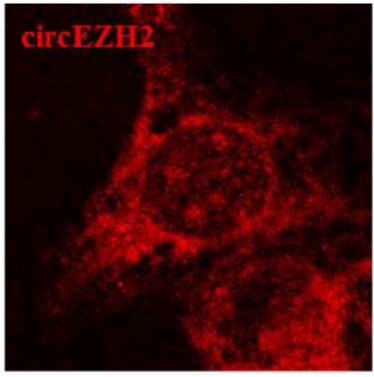

B

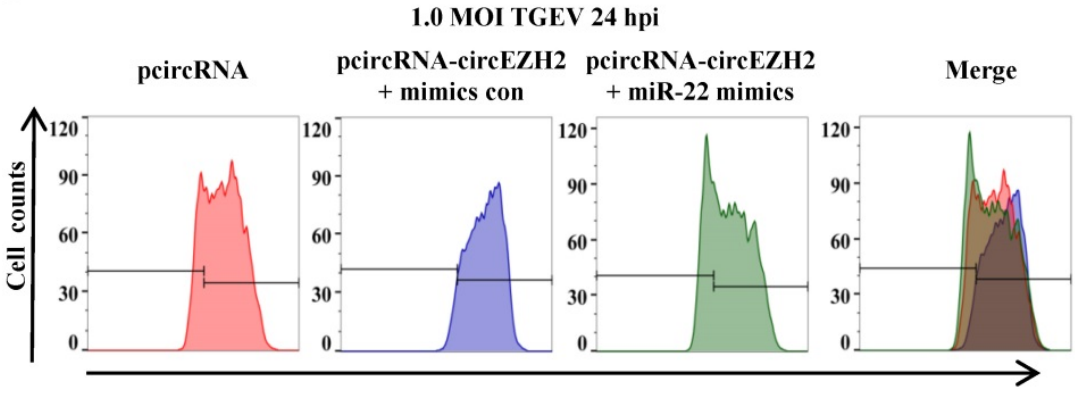

BBcell Probe ${ }^{\mathrm{TM}}$ M61 Fluorescence
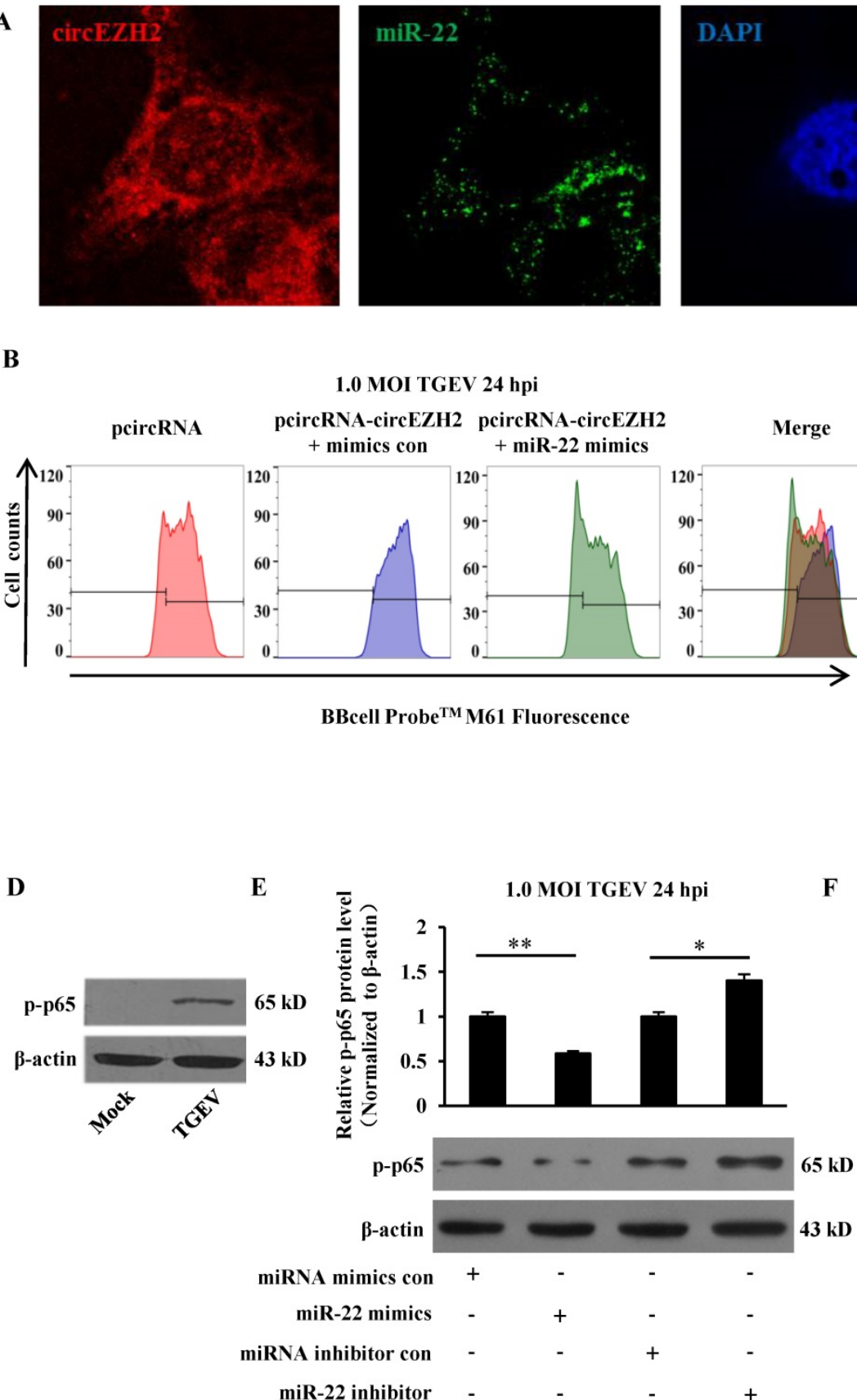

F
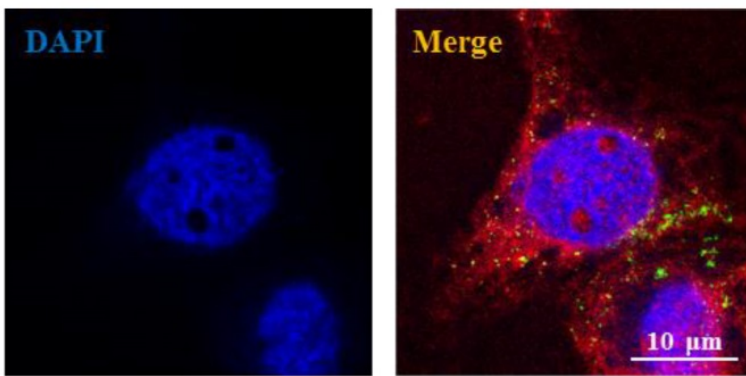

C
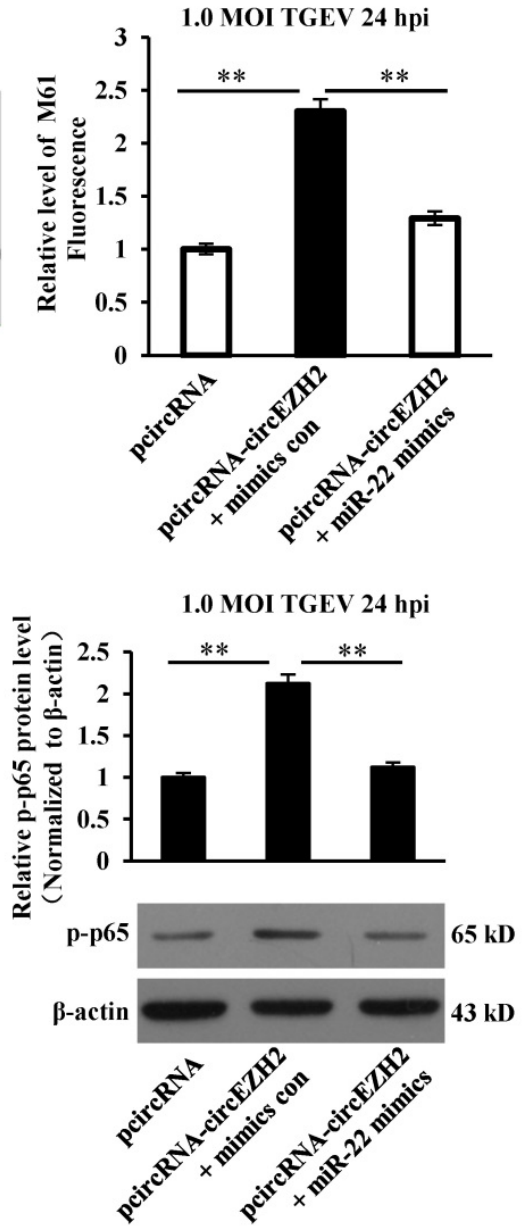

Figure 4 circEZH2 functions as sponge of miR-22. (A) Colocalization between miR-22 and circEZH2 in IPEC-J2 infected with TGEV. circEZH2 is shown in red. miR-22 is shown in green. Nucleus is shown in blue. (B) The combined effect of circEZH2 and miR-22 on TGEV-induced mPTP opening in IPEC-J2. (C) Quantification of mPTP opening level of Figure 4B using FACS. (D) The effect of TGEV on p-p65. (E) The effect of miR-22 on p-p65. (F) The combined effect of circEZH2 and miR-22 on P-p65. ** $p<0.01$ in comparison with the control. 
A

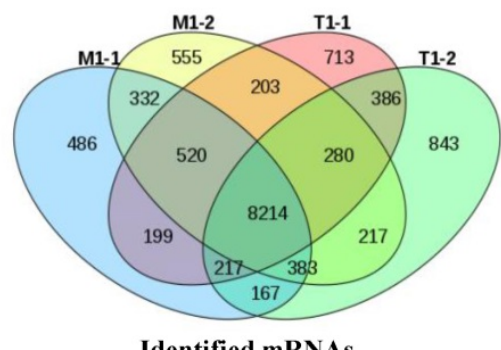

B

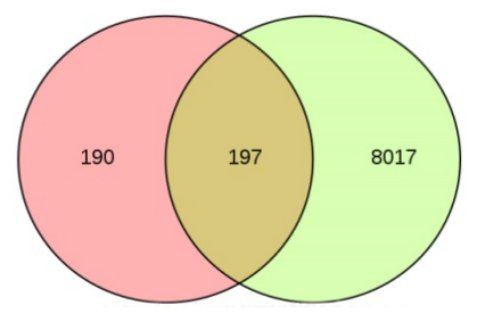

Targets of miR-22 Identified mRNAs

D

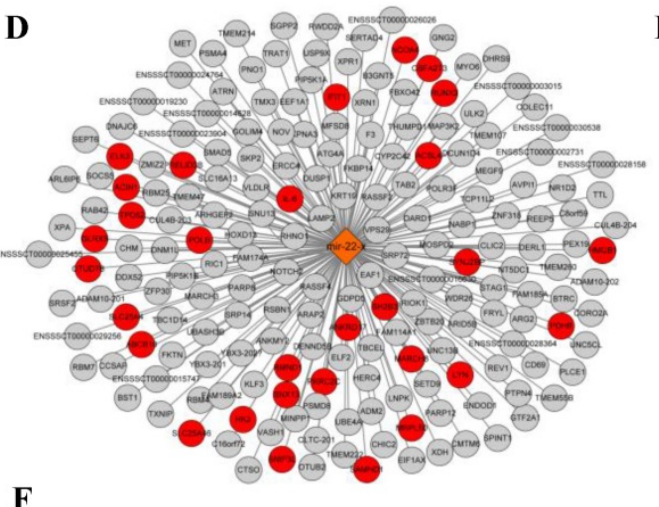

F

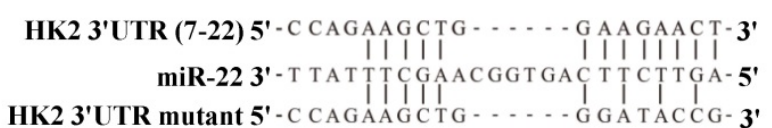

G

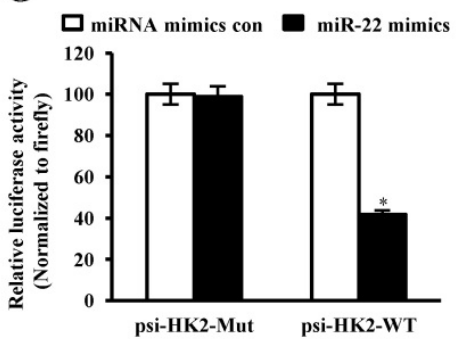

H

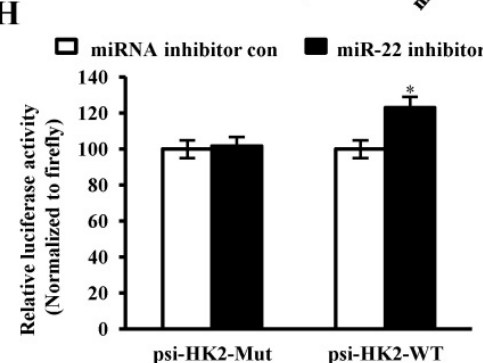

C

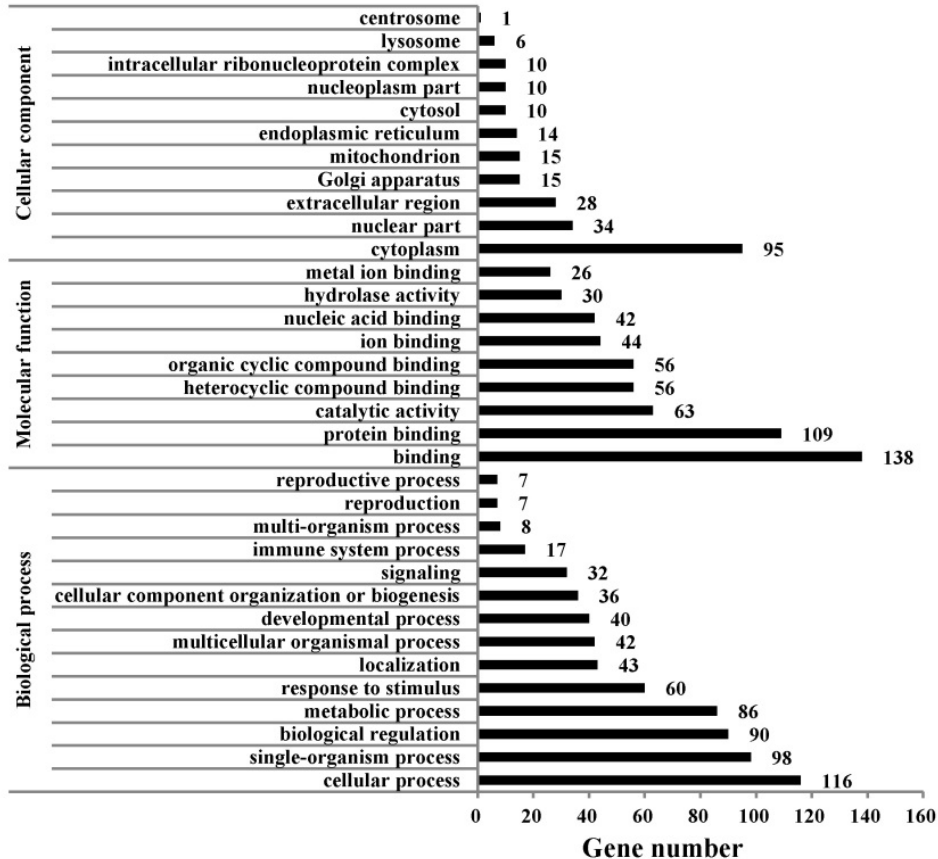

$\mathbf{E}$

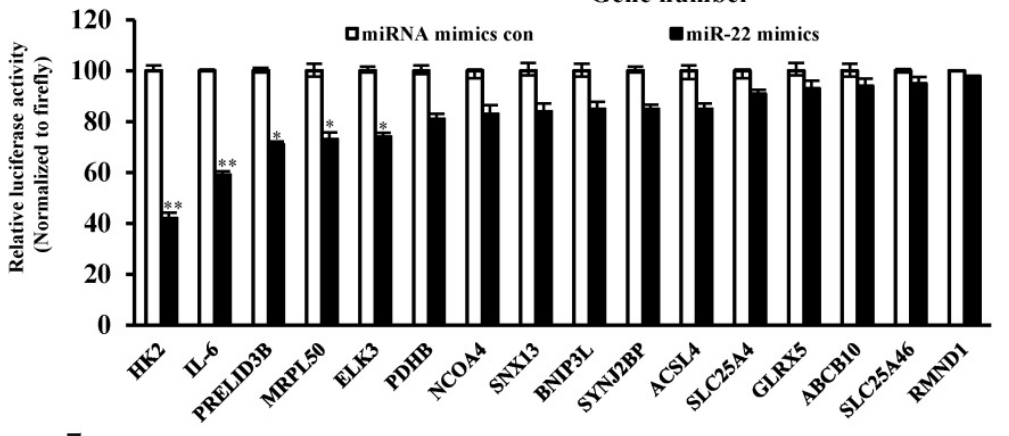

,

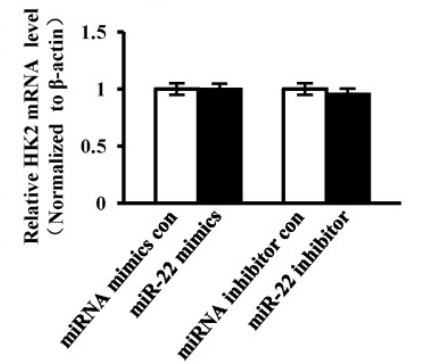

$\mathbf{J}$
$\mathrm{HK} 2--\longrightarrow 102 \mathrm{kD}$

$\beta$-actin $43 \mathrm{kD}$
miRNA mimics con

miR-22 mimics

miRNA inhibitor con

miR-22 inhibitor

Figure 5 Correlation analysis between miR-22 target genes and identified mRNAs. (A) Venn diagram showed the intersection of mRNAs among the four samples. (B) Venn diagram showed the intersection between miR-22 targets and overlapped mRNAs. (C) GO enrichment analysis of miR-22 target genes. (D) The interaction network of miR-22 and its target genes. Red circles indicate target genes of miR-22 localized at mitochondria or participated in the immune system process. (E) The binding ability of miR-22 to 3' UTRs of 16 potential target genes. (F) Schematic overview of mutation of swine HK2 3' UTR sequence. The upper sequence is the binding site of miR-22 in 3' UTR of swine HK2. The middle is the sequence of mature miR-22. The lower sequence is the mutated binding site sequence of miR-22 of HK2 3 ' UTR. (G) and (H) The relative luciferase activities of psi-HK2-WT and psi-HK2-Mut mediated by miR-22 mimics and miR-22 inhibitor. (I) The relative mRNA level of HK2 in IPEC-J2 treated with miR-22 mimics and miR-22 inhibitor. (J) The effect of miR-22 on expression of HK2. $* p<0.05$ and $* * p<0.01$ in comparison with the control. 


\section{si-HK2 had no effect on NF-KB activation}

Previous studies suggested that circEZH2 potentiated TGEV-induced activation of NF-kB pathway through attaching to miR-22. To investigate the effect of HK2 on TGEV-induced NF-KB activation,
IPEC-J2 cells were transfected with si-HK2 or Negetive Control (NC siRNA) and subsequently infected with TGEV at $1 \mathrm{MOI}$ for $24 \mathrm{~h}$. The result showed that p-p65 protein level was not affected by siHK2 (Figure 6F).
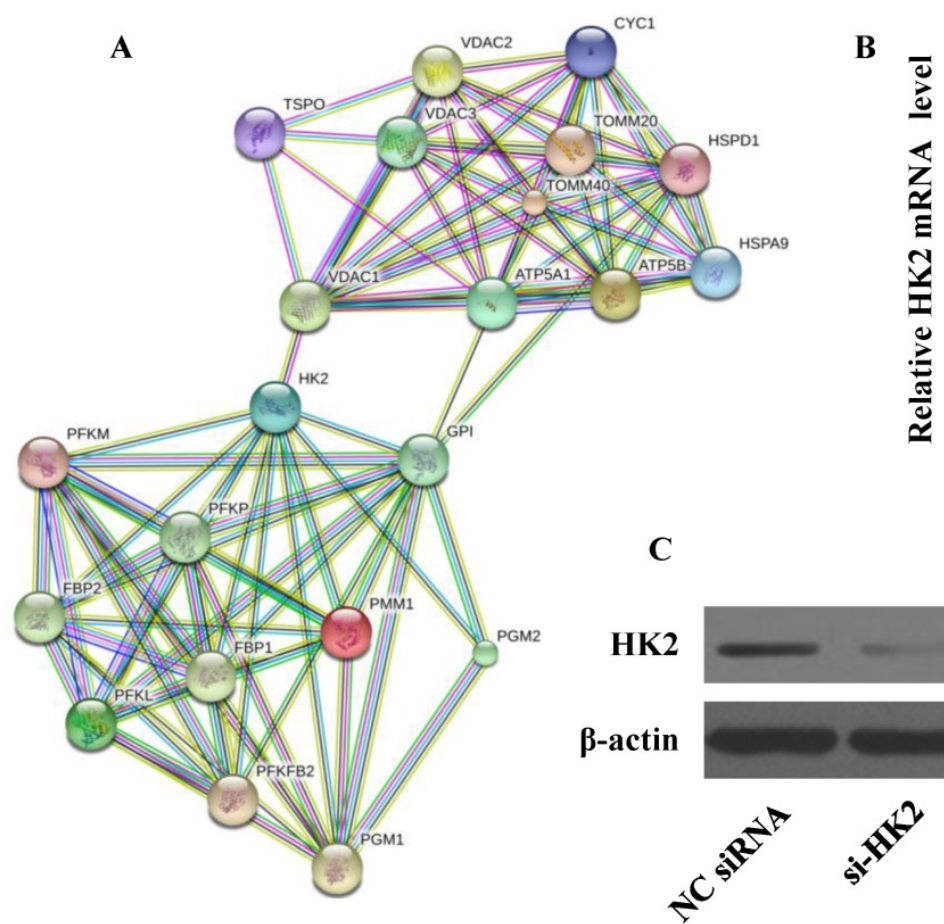

D

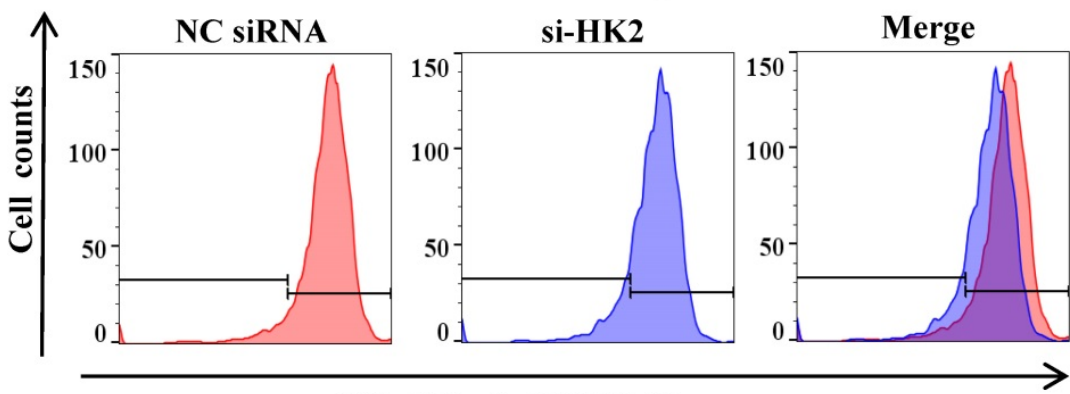

BBcell Probe ${ }^{\mathrm{TM}}$ M61 Fluorescence
B

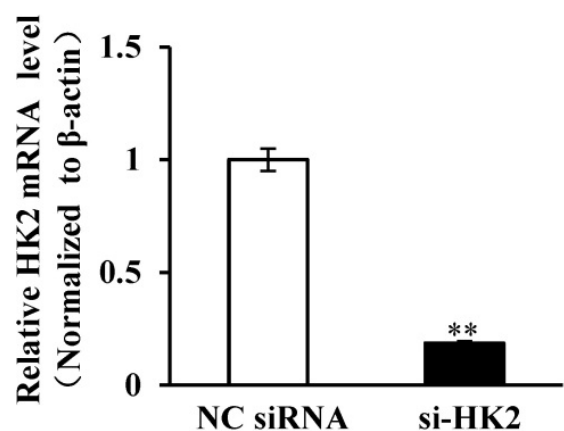

$65 \mathrm{kD}$

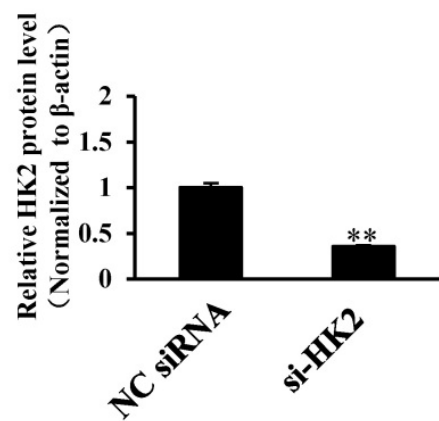

E

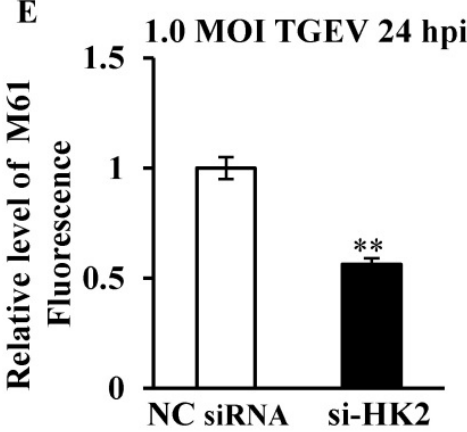

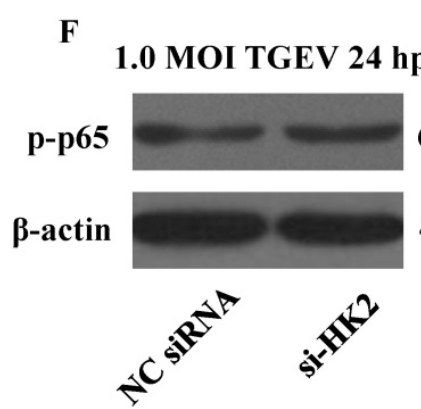

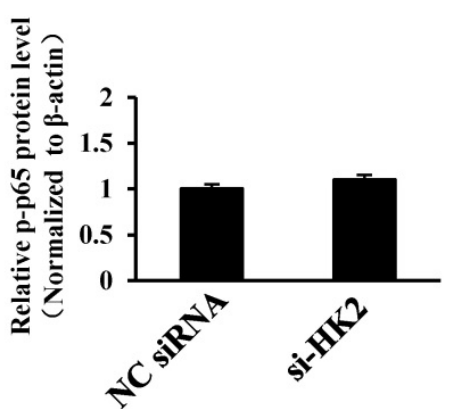

Figure 6 The effects of HK2 on mPTP opening and NF-KB pathway. (A) Network node represents protein. Colored nodes represent the query proteins and the first shell of interactors. White nodes represent the second shell of interactions. Small nodes are the proteins with unknown 3D structure. Large nodes are the proteins with known or predicted 3D structure. Lines represent protein-protein interactions. Light blue line represents from curated databases. Purple line represents experimentally determined. Green line represents gene neighborhood. Red line represents gene fusions. Navy blue line represents gene co-occurrence. Yellow represents textmining. Black line represents co-expression. Grey line represents protein homology. (B) and (C) The silencing effect of si-HK2. (D) The effect of si-HK2 on TGEV-induced mPTP opening. (E) Quantification of mPTP opening level of Figure 6D. (F) The effect of siHK2 on p-p65. $* * p<0.01$ in comparison with the control. 


\section{miR-22 promoted $\mathrm{mPTP}$ opening through suppressed NF-KB pathway and targeting IL-6}

IL-6 is a key mediator in response to acute inflammation caused by viral infection, including TGEV, PEDV, HSV, H1N1 influenza virus, and HBV[30-33]. Previous studies have confirmed that IL-6 was involved in regulating the NF-KB pathway[34]. To determine the direct interaction between miR-22 and $3^{\prime}$ UTR of IL-6 mRNA, the porcine IL-6 3' UTR or its mutant was respectively cloned into the 3' UTR of a Renilla luciferase gene in a luciferase reporter psiCHECK-2 (Figure 7A). The constructs and miR-22 mimics (or miRNA mimics control, miR-22 inhibitor, miRNA inhibitor control) were co-transfected into IPEC-J2 cells. Compared with the control, introduction of exogenous miR-22 decreased the psi-IL-6-WT reporter activity by $62 \%$, while overexpression and knockdown of miR-22 using miR-22 mimics and inhibitor did not affect psi-IL-6-Mut reporter activity (Figure 7B and C). To determine whether miR-22 inhibited IL-6 expression on mRNA level, miR-22 mimics (or miRNA mimics control, miR-22 inhibitor, miRNA inhibitor control) was transfected into IPEC-J2 cells. The mRNA level of IL-6 was assessed by real-time PCR. Introduction of exogenous miR-22 decreased mRNA level of IL-6 in IPEC-J2 cells. Knockdown of endogenous miR-22 using miR-22 inhibitor increased the mRNA level of IL-6 (Figure 7D).To investigate the effect of IL-6 on NF-kB pathway, IPEC-J2 were transfected with si-IL-6 or NC siRNA, and subsequently infected with TGEV at $1 \mathrm{MOI}$ for $24 \mathrm{~h}$. The results showed that p-p65 significantly decreased at protein level by si-IL-6 (Figure 7E and F). To examine whether miR-22 suppressed NF-kB pathway via interacting with IL-6, miR-22 inhibitor and the si-IL-6 were co-transfected into IPEC-J2. The results revealed that p-p65 significantly down-regulated in IPEC-J2 by miR-22 inhibitor and si-IL-6 co-transfection in comparison with miR-22 inhibitor alone (Figure 7G). To investigate the effect of IL-6 on mPTP opening, IPEC-J2 cells were transfected with si-IL-6 or NC siRNA, and subsequently infected with TGEV at 1 MOI for $24 \mathrm{~h}$. The level of MPTP opening was detected using flow cytometry. The results showed that fluorescence intensity for BBcellProbeTM M61 significantly decreased by si-IL-6 (Figure $7 \mathrm{H}$ and I), indicating that IL-6 decreased TGEV-induced mPTP opening. IPEC-J2 cells were pretreated with $10 \mu \mathrm{M}$ NF- $\mathrm{kB}$ inhibitor (BAY 11-7082) for $60 \mathrm{~min}$ and subsequently infected with TGEV at $1 \mathrm{MOI}$ for $24 \mathrm{~h}$. The level of MPTP opening was detected using flow cytometry. The results showed that fluorescence intensity for BBcellProbeTM M61 significantly decreased by BAY 11-7082 (NF-kB inhibitor) (Figure 7J-L).

\section{Discussion}

IPEC are the target cells for TGEV and contain rich mitochondria contributing to absorption of nutrition and immune response for pathogen infection[35]. TGEV infection causes mitochondrial damage[36-38], which can subsequently result in various pathological processes, including inflammation, cell death. TGEV infection can cause inflammatory response in IPEC-J2 and activate NF-kB pathway [21].

mPTP is the switch of mitochondrial damage and is regulated by NF-KB pathway[27, 39]. $\mathrm{mPTP}$ is a channel that spans from the outer mitochondrial membrane to the mitochondrial matrix and controls the migration of molecules into and out of mitochondria[40]. Persistent opening of mPTP in response to high concentration of $\mathrm{Ca}^{2+}$ can lead to decrease of the mitochondrial membrane potential $(\triangle \psi \mathrm{m})$ and makes mitochondria become permeable to molecules less than $1.5 \mathrm{kDa}$ and water [11, 12, 41, 42]. Therefore, the opening of mPTP is the key link of mitochondrial damage. Mitochondrial damage can trigger inflammation response [43, 44]. We previously reported that circEZH2 activated NF-KB pathway, indicating circEZH2 regulating TGEV-caused inflammation response via NF-kB pathway[21]. It was recently reported that TGEV non-structural protein 2 (nsp2) promotes inflammation via NF-KB pathway[45]. Therefore, TGEV nsp2 may contribute to inflammation via regulating circEZH2. NF-KB pathway is a key pathway that regulates inflammation restrains mitochondrial damage and cell death via suppressing $\mathrm{MPTP}$ opening in ventrucular myocytes [27], correlating with our finding that inhibition of NF-kB pathway promoted TGEV-induced mPTP opening.

circRNAs are a class of non-coding RNA and play important roles in many biological and pathological processes, including development, tumorigenesis, viral infection, inflammation via sponging with miRNAs, interacting with proteins, regulating transcription and translation of genes, and regulating parental genes $[46,47]$. Here, we found that circEZH2 regulated $\mathrm{mPTP}$ opening via binding miR-22, while not affecting its parental gene, EZH2. Therefore, it will be of interest to investigate whether circEZH2 play regulatory roles through interacting with proteins or modulating gene transcription or translation. 
A

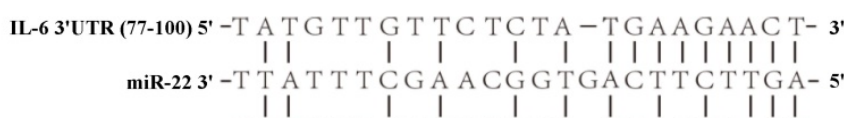
IL-6 3'UTR mutant 5' -T A T G T T G T T C T C T A - T T ACGCAC T- 3'

B

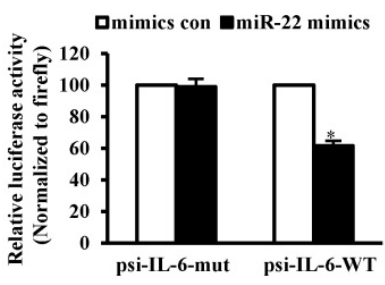

C

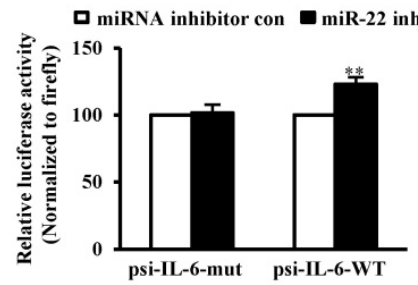

D

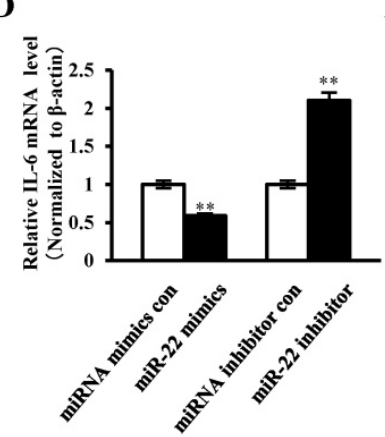

$\mathbf{E}$

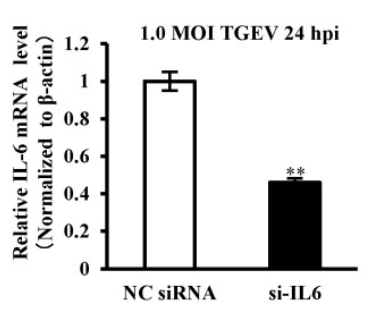

F

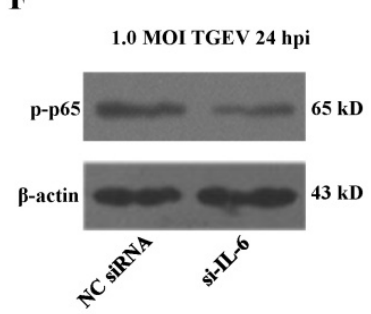

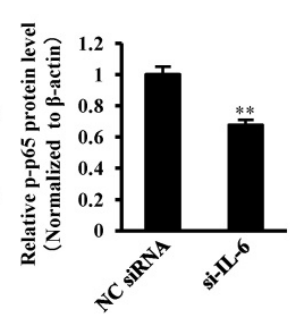

G
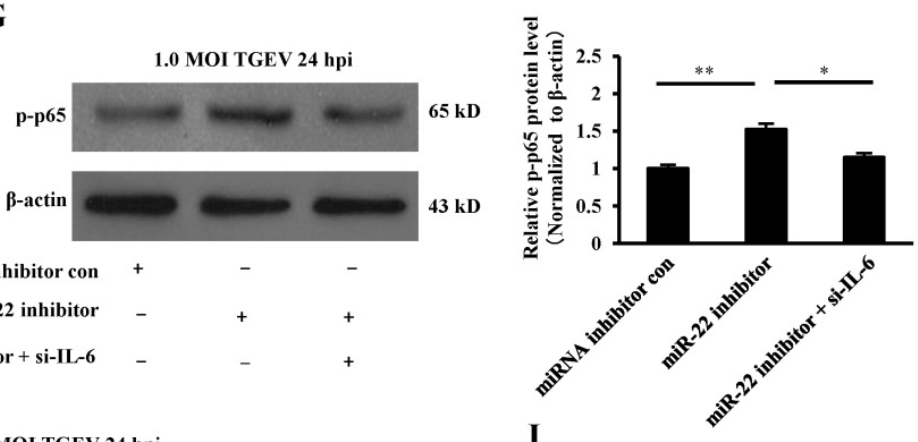

I

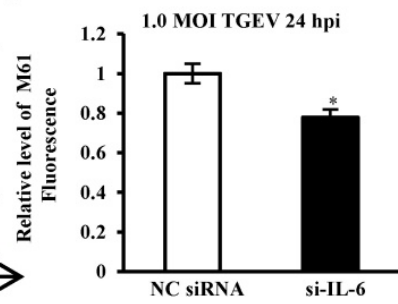

si-IL-6

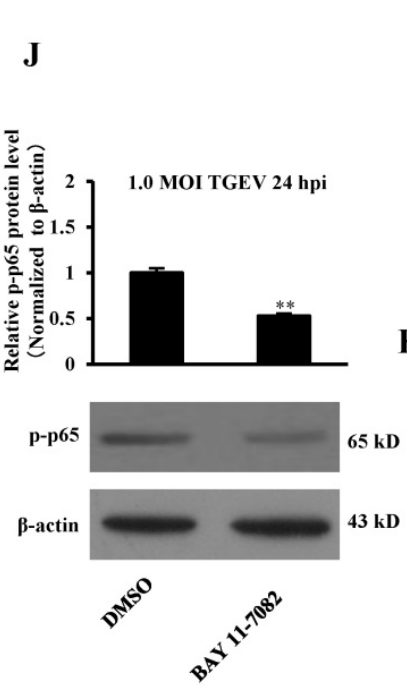

H
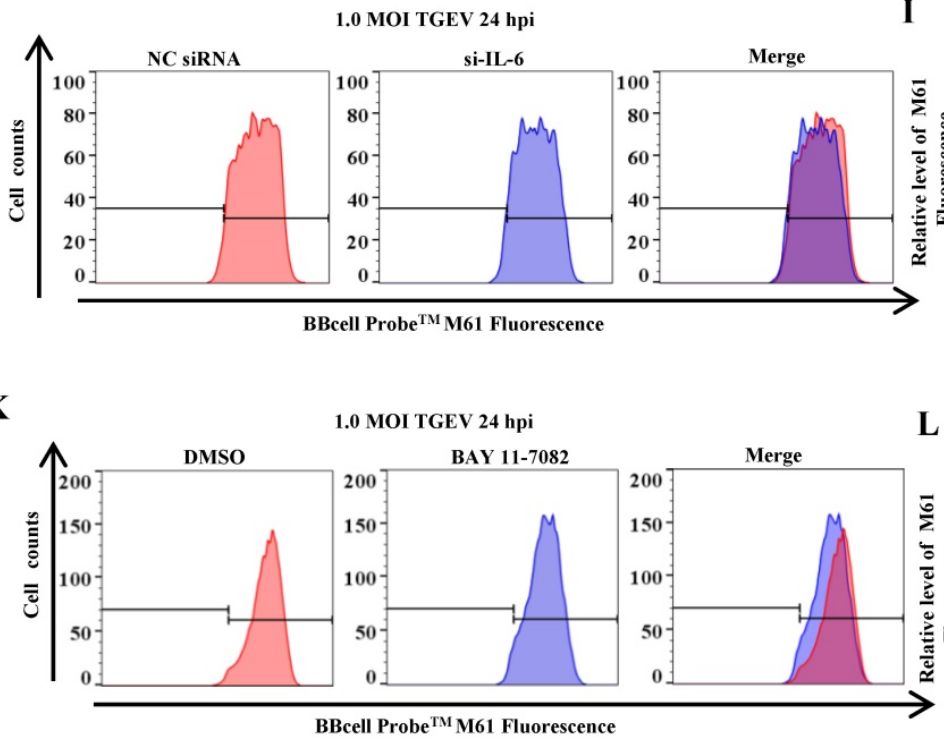

L $\quad 1.0$ MOI TGEV $24 \mathrm{hpi}$

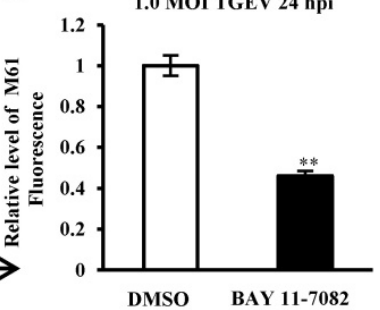

Figure 7 miR-22 promoted TGEV-induced mPTP opening through suppressing NF-KB pathway and targeting IL-6. (A) Schematic overview of mutation of swine IL-6 3' UTR sequence. The upper sequence is the binding site of miR-22 in 3' UTR of swine IL-6. The middle is the sequence of mature miR-22. The lower sequence is the mutated sequence of IL-6 3' UTR. (B) and (C) The relative luciferase activities of psi-IL-6-WT and psi-IL-6-Mut mediated by miR-22 mimics and miR-22 inhibitor. (D) The relative mRNA level of IL- 6 in IPEC-J2 treated with miR-22 mimics and miR-22 inhibitor. $* p<0.05$ in comparison with the control. ** $p<0.01$ in comparison with the control. (E) Silencing effect of si-IL-6. (F) The effect of si-IL-6 on P-p65. (G) The combined effects of miR-22 inhibitor and si-IL-6 on P-p65. (H) The effect of si-IL-6 on TGEV-induced mPTP opening. (I) Quantification of mPTP opening level of Figure 7H. (J) The effect of BAY 11-7082 (NF-KB inhibitor s) on P-p65. (K) The effect of BAY 11-7082 on TGEV-induced mPTP opening. (L) Quantification of mPTP opening level of Figure 7K. ${ }^{*} \mathrm{p}<0.05$ in comparison with the control. $* * p<0.01$ in comparison with the control.

Voltage-dependent anion channel 1 (VDAC1) is an important regulator of $\mathrm{mPTP}$ opening. HK2 interacts with VDAC1 to change the conformation of VDAC1, which leads to an inhibition of mPTP opening [48-50]. This correlates with our discovery that silencing HK2, the target of miR-22, aggravates
TGEV-induced mPTP opening. However, HK2 does not regulate NK-kB activation. Not only HK2, IL-6 was also identified as the target of miR-22 and suppressed TGEV-induced $\mathrm{MPTP}$ opening via activating NF-kB pathway, suggesting that miR-22 regulates TGEV-induced $\mathrm{mPTP}$ opening via at least 
two pathways. Our finding is supported by the discovery that IL-6 reduces hyperoxia-induced mitochondrial damage via Bcl-2-induced Bak-mitofusions interaction and activates NF-KB pathway through STAT3 pathway[34, 51] and provides a new mechanism of miR-22 in mitochondrial function.

In summary, circEZH2 presents a novel function in activating NF- $\mathrm{KB}$ and regulating $\mathrm{MPTP}$ opening via binding miR-22 and provides a potential novel pharmacologic target in the interventional approach to prevent and control TGEV infection.

\section{Abbreviations}

TGE: transmissible gastroenteritis; TGEV: transmissible gastroenteritis coronavirus; IPEC: porcine intestinal epthelial cell; $\mathrm{mPTP}$ : mitochondrial permeability transition pore; NF-KB: nuclear factor kappa-light-chain-enhancer of activated B cells; HK2: Hexokinase 2; IL-6: interleukin 6; CoVs: Coronaviruses; ARDS: acute respiratory distress syndrome; TSCC: tongue squamous cell carcinoma; IRF3: interferon regulating factor 3; HBx: Hepatitis B virus $x$ protein; PEDV: porcine epidemic diarrhea virus; PoRV: Porcine Rotavirus; PCV2: porcine circovirus type 2; PBS: phosphate-buffered saline; HE: hematoxylin-eosin; HRP: Horseradish peroxidase; DMEM: Dulbecco's Minimal Essential Medium; PVDF: polyvinylidene difluoride; ECL: enhanced chemiluminescence; MOI: multiplicity of infection; DAPI: 4,6-diamidino-2-phenylindole; PMSF: phenylmethyl sulonylfluoride; FISH: Fluorescence in situ hybridization; FACS: Fluorescence Activated Cell Sorting.

\section{Supplementary Material}

Supplementary figures.

http://www.ijbs.com/v15p2051s1.pdf

Table S1. http:/ /www.ijbs.com/v15p2051s2.xlsx

Table S2. http://www.ijbs.com/v15p2051s3.xlsx

Table S3. http://www.ijbs.com/v15p2051s4.xlsx

Table S4. http://www.ijbs.com/v15p2051s5.xlsx

\section{Acknowledgements}

This work was supported by Key Research and Development Project in Shaanxi Province (Grant No. 2018ZDXM-NY-064), National Natural Science Foundation of China (Grant No. 31472167), Science and Technology Planning Project of Yangling demonstration zone (Grant No. 2018NY-35), and Central Project of Major Agricultural Technology Promotion Funds (Grant No. K3360217060).

\section{Authorship}

Xiaomin Zhao and Xuelian Ma designed the experiments, interpreted the data and wrote the article. Jianxiong Guo was involved in drafting and revising the manuscript. $\mathrm{Mi} \mathrm{Mi}$ and Kaili Wang performed the experiments and analyzed the data. Chuyi Zhang and Xiaoyi Tang were involved in drafting the manuscript and prepared figures and/or tables. Lingling Chang and Yong Huang involved in revising the manuscript. Dewen Tong conceived and designed the experiments, contributed reagents/materials/analysis tools, reviewed drafts of the paper. All authors have read the manuscript and approved to submit it to this journal.

\section{Ethics approval}

All animal procedures were conducted following the guidelines of the Animal Ethics Committee at Northwest A\&F University under the document 2011-31101684. The sampling procedures complied with the "Guidelines on Ethical Treatment of Experimental Animals" (2006) Number 398 set by the Ministry of Science and Technology, China. The procedures in the present study had received prior approval from the Experimental Animal Manage Committee of Northwest A\&F University (Approval ID: 20160612).

\section{Competing Interests}

The authors have declared that no competing interest exists.

\section{References}

1. Chen IY, Moriyama M, Chang MF, Ichinohe T. Severe Acute Respiratory Syndrome Coronavirus Viroporin 3a Activates the NLRP3 Inflammasome. Frontiers in microbiology. 2019; 10: 50.

2. Faridi U. Middle East respiratory syndrome coronavirus (MERS-CoV): Impact on Saudi Arabia, 2015. Saudi journal of biological sciences. 2018; 25: 1402-5.

3. Walls AC, Xiong X, Park YJ, Tortorici MA, Snijder J, Quispe J, et al. Unexpected Receptor Functional Mimicry Elucidates Activation of Coronavirus Fusion. Cell. 2019; 176: 1026-39 e15.

4. Cui T, Theuns S, Desmarets LMB, Xie J, De Gryse GMA, Yang B, et al. Establishment of porcine enterocyte/myofibroblast co-cultures for the growth of porcine rota- and coronaviruses. Scientific reports. 2018; 8: 15195.

5. Niederwerder MC, Hesse RA. Swine enteric coronavirus disease: A review of 4 years with porcine epidemic diarrhoea virus and porcine deltacoronavirus in the United States and Canada. Transboundary and emerging diseases. 2018; 65: 660-75.

6. Yang Z, Ran L, Yuan P, Yang Y, Wang K, Xie L, et al. EGFR as a Negative Regulatory Protein Adjusts the Activity and Mobility of NHE3 in the Cell Membrane of IPEC-J2 Cells With TGEV Infection. Frontiers in microbiology. 2018; 9: 2734.

7. Zhang S, Hu W, Yuan L, Yang Q. Transferrin receptor 1 is a supplementary receptor that assists transmissible gastroenteritis virus entry into porcine intestinal epithelium. Cell communication and signaling : CCS. 2018; 16: 69.

8. Brown D, Breton S. Mitochondria-rich, proton-secreting epithelial cells. The Journal of experimental biology. 1996; 199: 2345-58.

9. Saif LJ. Mucosal immunity: an overview and studies of enteric and respiratory coronavirus infections in a swine model of enteric disease. Veterinary immunology and immunopathology. 1996; 54: 163-9.

10. Zhou T, Zhu M, Liang Z. (-)-Epigallocatechin-3-gallate modulates peripheral immunity in the MPTP-induced mouse model of Parkinson's disease. Molecular medicine reports. 2018.

11. Liu P, Dong J. Protective effects of carnosic acid against mitochondria-mediated injury in $\mathrm{H} 9 \mathrm{c} 2$ cardiomyocytes induced by hypoxia/reoxygenation. Experimental and therapeutic medicine. 2017; 14: 5629-34. 
12. Wang $\mathrm{H}$, Chen $\mathrm{Y}$, Zhai N, Chen $\mathrm{X}$, Gan F, Li H, et al. Ochratoxin A-Induced Apoptosis of IPEC-J2 Cells through ROS-Mediated Mitochondrial Permeability Transition Pore Opening Pathway. Journal of agricultural and food chemistry. 2017; 65: 10630-7.

13. Zandalinas SI, Mittler R. ROS-induced ROS release in plant and animal cells. Free radical biology \& medicine. 2017

14. Yang J, Huo T, Zhang X, Ma J, Wang Y, Dong F, et al. Oxidative stress and cell cycle arrest induced by short-term exposure to dustfall PM2.5 in A549 cells. Environmental science and pollution research international. 2017.

15. El-Amine R, Germini D, Zakharova VV, Tsfasman T, Sheval EV, Louzada RAN, et al. HIV-1 Tat protein induces DNA damage in human peripheral blood B-lymphocytes via mitochondrial ROS production. Redox biology. 2017; 15: 97-108.

16. Reshi L, Wu HC, Wu JL, Wang HV, Hong JR. GSIV serine/threonine kinase can induce apoptotic cell death via p53 and pro-apoptotic gene Bax upregulation in fish cells. Apoptosis : an international journal on programmed cell death. 2016; 21: 443-58.

17. Lin $X$, Wang $R$, Zou $W$, Sun $X$, Liu X, Zhao L, et al. The Influenza Virus H5N1 Infection Can Induce ROS Production for Viral Replication and Host Cell Death in A549 Cells Modulated by Human Cu/Zn Superoxide Dismutase (SOD1) Overexpression. Viruses. 2016; 8.

18. Ivanov AV, Smirnova OA, Petrushanko IY, Ivanova ON, Karpenko IL, Alekseeva E, et al. HCV core protein uses multiple mechanisms to induce oxidative stress in human hepatoma Huh7 cells. Viruses. 2015; 7: 2745-70.

19. Zhu L, Mou C, Yang X, Lin J, Yang Q. Mitophagy in TGEV infection counteracts oxidative stress and apoptosis. Oncotarget. 2016.

20. Ding L, Xu X, Huang Y, Li Z, Zhang K, Chen G, et al. Transmissible gastroenteritis virus infection induces apoptosis through FasL- and mitochondria-mediated pathways. Veterinary microbiology. 2012; 158: 12-22.

21. Ma X, Zhao X, Zhang Z, Guo J, Guan L, Li J, et al. Differentially expressed non-coding RNAs induced by transmissible gastroenteritis virus potentially regulate inflammation and NF-kappaB pathway in porcine intestinal epithelial cell line. BMC genomics. 2018; 19: 747.

22. Gu $\mathrm{Y}$, Liu $\mathrm{H}$, Kong $\mathrm{F}$, Ye J, Jia $\mathrm{X}$, Zhang $\mathrm{Z}$, et al. miR-22/KAT6B axis is a chemotherapeutic determiner via regulation of PI3k-Akt-NF-kB pathway in tongue squamous cell carcinoma. Journal of experimental \& clinical cancer research : CR. 2018; 37: 164

23. Polioudakis D, Bhinge AA, Killion PJ, Lee BK, Abell NS, Iyer VR. A Myc-microRNA network promotes exit from quiescence by suppressing the interferon response and cell-cycle arrest genes. Nucleic acids research. 2013; 41: 2239-54.

24. Du JK, Cong BH, Yu Q, Wang H, Wang L, Wang CN, et al. Upregulation of microRNA-22 contributes to myocardial ischemia-reperfusion injury by interfering with the mitochondrial function. Free radical biology \& medicine. 2016; 96: 406-17.

25. Clippinger AJ, Gearhart TL, Bouchard MJ. Hepatitis B virus X protein modulates apoptosis in primary rat hepatocytes by regulating both NF-kappaB and the mitochondrial permeability transition pore. Journal of virology. 2009; 83: 4718-31.

26. Zhang C, Jiang $H$, Wang $P$, Liu $H$, Sun X. Transcription factor NF-kappa B represses ANT1 transcription and leads to mitochondrial dysfunctions. Scientific reports. 2017; 7: 44708.

27. Baetz D, Regula KM, Ens K, Shaw J, Kothari S, Yurkova N, et al. Nuclear factor-kappaB-mediated cell survival involves transcriptional silencing of the mitochondrial death gene BNIP3 in ventricular myocytes. Circulation. 2005; 112: 3777-85.

28. Song X, Zhao X, Huang $Y$, Xiang H, Zhang W, Tong D. Transmissible gastroenteritis virus (TGEV) infection alters the expression of cellular microRNA species that affect transcription of TGEV gene 7. International journal of biological sciences. 2015; 11: 913-22.

29. Heckman KL, Pease LR. Gene splicing and mutagenesis by PCR-driven overlap extension. Nature protocols. 2007; 2: 924-32.

30. Aoki R, Kawamura T, Goshima F, Ogawa Y, Nakae S, Nakao A, et al. Mast cells play a key role in host defense against herpes simplex virus infection through TNF-alpha and IL-6 production. The Journal of investigative dermatology. 2013; 133: 2170-9.

31. Chiaretti A, Pulitano S, Barone G, Ferrara P, Romano V, Capozzi D, et al. IL-1 beta and IL-6 upregulation in children with H1N1 influenza virus infection. Mediators of inflammation. 2013; 2013: 495848.

32. Quetier I, Brezillon N, Duriez M, Massinet H, Giang E, Ahodantin J, et al. Hepatitis B virus $\mathrm{HBx}$ protein impairs liver regeneration through enhanced expression of IL-6 in transgenic mice. Journal of hepatology. 2013; 59: 285-91.

33. Huan CC, Wang HX, Sheng XX, Wang R, Wang X, Liao Y, et al. Porcine epidemic diarrhea virus nucleoprotein contributes to HMGB1 transcription and release by interacting with C/EBP-beta. Oncotarget. 2016; 7: 75064-80.

34. Hartman ZC, Poage GM, den Hollander P, Tsimelzon A, Hill J, Panupinthu N, et al. Growth of triple-negative breast cancer cells relies upon coordinate autocrine expression of the proinflammatory cytokines IL-6 and IL-8. Cancer Res. 2013; 73: 3470-80.

35. Elbrond VS, Jones CJ, Skadhauge E. Localization, morphology and function of the mitochondria-rich cells in relation to transepithelial $\mathrm{Na}(+)$-transport in chicken lower intestine (coprodeum). Comparative biochemistry and physiology Part A, Molecular \& integrative physiology. 2004; 137: 683-96.
36. Ding L, Zhao X, Huang Y, Du O, Dong F, Zhang H, et al. Regulation of ROS in transmissible gastroenteritis virus-activated apoptotic signaling. Biochemical and biophysical research communications. 2013; 442: 33-7.

37. Zhao X, Bai X, Guan L, Li J, Song X, Ma X, et al. microRNA-4331 Promotes Transmissible Gastroenteritis Virus (TGEV)-induced Mitochondrial Damage Via Targeting RB1, Upregulating Interleukin-1 Receptor Accessory Protein (IL1RAP), and Activating p38 MAPK Pathway In Vitro. Molecular \& cellular proteomics : MCP. 2018; 17: 190-204

38. Zhao X, Song X, Bai X, Tan Z, Ma X, Guo J, et al. microRNA-222 Attenuates Mitochondrial Dysfunction During Transmissible Gastroenteritis Virus Infection. Molecular \& cellular proteomics : MCP. 2019; 18: 51-64.

39. Warnsmann V, Meyer N, Hamann A, Kogel D, Osiewacz HD. A novel role of the mitochondrial permeability transition pore in (-)-gossypol-induced mitochondrial dysfunction. Mechanisms of ageing and development. 2018; 170: $45-58$

40. Briston T, Selwood DL, Szabadkai G, Duchen MR. Mitochondrial Permeability Transition: A Molecular Lesion with Multiple Drug Targets. Trends in pharmacological sciences. 2019; 40: 50-70.

41. Yang J, Huo T, Zhang X, Ma J, Wang Y, Dong F, et al. Oxidative stress and cell cycle arrest induced by short-term exposure to dustfall PM2.5 in A549 cells. Environmental science and pollution research international. 2018; 25: 22408-19.

42. Zandalinas SI, Mittler R. ROS-induced ROS release in plant and animal cells. Free radical biology \& medicine. 2018; 122: 21-7.

43. Valcarcel-Ares MN, Riveiro-Naveira RR, Vaamonde-Garcia C, Loureiro I, Hermida-Carballo L, Blanco FJ, et al. Mitochondrial dysfunction promotes and aggravates the inflammatory response in normal human synoviocytes. Rheumatology (Oxford, England). 2014; 53: 1332-43.

44. Dela Cruz CS, Kang MJ. Mitochondrial dysfunction and damage associated molecular patterns (DAMPs) in chronic inflammatory diseases. Mitochondrion. 2018; 41: 37-44.

45. Wang L, Qiao X, Zhang S, Qin Y, Guo T, Hao Z, et al. Porcine transmissible gastroenteritis virus nonstructural protein 2 contributes to inflammation via NF-kappaB activation. Virulence. 2018; 9: 1685-98.

46. Chen YG, Kim MV, Chen X, Batista PJ, Aoyama S, Wilusz JE, et al. Sensing Self and Foreign Circular RNAs by Intron Identity. Molecular cell. 2017; 67: 228-38 e5.

47. Liang D, Tatomer DC, Luo Z, Wu H, Yang L, Chen LL, et al. The Output of Protein-Coding Genes Shifts to Circular RNAs When the Pre-mRNA Processing Machinery Is Limiting. Molecular cell. 2017; 68: 940-54 e3.

48. Xue YN, Yu BB, Li JL, Guo R, Zhang LC, Sun LK, et al. Zinc and p53 disrupt mitochondrial binding of HK2 by phosphorylating VDAC1. Experimental cell research 2019: 374: 249-58.

49. Zhang D, Yip YM, Li L. In silico construction of HK2-VDAC1 complex and investigating the HK2 binding-induced molecular gating mechanism of VDAC1. Mitochondrion. 2016; 30: 222-8.

50. Zhou $\mathrm{H}, \mathrm{Hu} \mathrm{S}$, Jin Q, Shi C, Zhang $\mathrm{Y}$, Zhu P, et al. Mff-Dependent Mitochondrial Fission Contributes to the Pathogenesis of Cardiac Microvasculature Ischemia/Reperfusion Injury via Induction of mROS-Mediated Cardiolipin Oxidation and HK2/VDAC1 Disassociation-Involved mPTP Opening. Journal of the American Heart Association. 2017; 6

51. Waxman AB, Kolliputi N. IL-6 protects against hyperoxia-induced mitochondrial damage via Bcl-2-induced Bak interactions with mitofusins. American journal of respiratory cell and molecular biology. 2009; 41: 385-96. 